\title{
LAS PERSONAS INTERSEXUALES Y EL DERECHO: POSIBLES RESPUESTAS JURÍDICAS PARA UN COLECTIVO INVISIBLE
}

\author{
Intersex people and the law. \\ A few answers for an invisible collective
}

\author{
ELENA LAUROBA LACASA \\ Universidad de Barcelona \\ e.lauroba@ub.edu
}

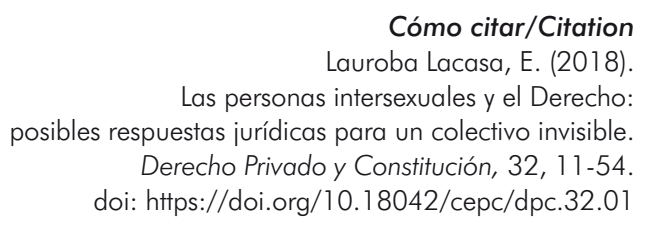

(Recepción: 02/05/2018. Aceptación tras revisión: 05/06/2018. Publicación: 22/06/2018.

\section{Resumen}

Se consideran intersexuales las personas que no pueden ser identificadas biológicamente como pertenecientes al género femenino o masculino dada la existencia de singularidades cromosómicas, hormonales y/o anatómicas. Tradicionalmente esta situación se ha zanjado con la adscripción temprana a uno de los dos sexos, con el soporte de intervenciones quirúrgicas, tratamientos hormonales y secretismo. Dichas acciones suponen una lesión de los derechos fundamentales de los intersexuales, pero

1 Este estudio se benefició de la beca de bioética de la Fundación Víctor Grifols i Lucas 2015, a la que se agradecen la confianza depositada y las facilidades durante la realización del trabajo. 
se consideraba que redundaban en su mejor inserción social. A día de hoy, tras los estudios de significados organismos internacionales, la situación está cambiando desde una perspectiva médica, y se aboga por una demora en las operaciones. También se están buscando mecanismos para solventar la necesidad de inscribirlos al nacer, con el consiguiente debate sobre la admisión jurídica de un tercer sexo. Contribuyen a visibilizar a los intersexuales las acciones en contra de la discriminación del colectivo LGTB, al que se asocian, pese a las diferencias.

\title{
Palabras clave
}

Intersexuales; derechos fundamentales; identidad de género; consentimiento informado; discriminación.

\begin{abstract}
Intersex are defined as people who cannot be biologically identified as belonging to the feminine or masculine sex, given the existence of chromosomal, hormonal and / or anatomical singularities. The traditional solution to this problem has been to promptly assign one of the two sexes, with the support of urgent surgical interventions, hormonal treatments and secrecy. These actions amount to a violation of fundamental rights of intersex people, but it has been considered that they contributed to their social integration. Today, the situation is changing from a medical perspective, and there are calls to postpose surgery until minors can give their consent. Also, public institutions are developing mechanisms to ensure their safe registration after birth, thus opening the debate on the legal admission of a third sex. Intersex people are gaining visibility thanks to the anti-discrimination actions conducted by the LGBT community.
\end{abstract}

\section{Keywords}

Intersexual; people; fundamental rights; gender identity; informed consent; discrimination. 


\section{SUMARIO}

I. PRESENTACIÓN. II. LA CONDICIÓN DE INTERSEXUAL: 1. Un concepto marco 2. La denominación «identificativa» de las personas intersexuales. 3. Sexo, género, orientación sexual, identidad de género. 4. Los intersexuales como integrantes de la secuencia LGBT: ahora LGBTI. III. CLAVES PARA EL ANÁLISIS JURÍDICO: LAS RESOLUCIONES DE LA ASAMBLEA PARLAMENTARIA DEL CONSEJO DE EUROPA. IV. LAS OPERACIONES «CORRECTIVAS» TEMPRANAS: 1. La operación como emergencia: actuar «por el bien de todos». 2. Los derechos fundamentales que las operaciones lesionan. 3. ¿̇ué consentimiento y qué emisor en caso de optar por la operación quirúrgica?: 3. 1. Materiales para encarar el problema: las decisiones del TC de Colombia. 3.2. La intervención de los progenitores. 3.3. La asistencia de terceros. 3.4. La participación (consentimiento) del menor. 4. El asesoramiento continuado de un equipo pluridisciplinar. 5. La regulación de Malta. V. LA INSCRIPCIÓN EN LOS REGISTROS i̇ES FACTIBLE (Y BENÉFICO) SUPERAR LA CLASIFICACIÓN BINARIA EN EL ÁMBITO ADMINISTRATIVO?: 1. El refrendo registral urgente. 2. La regulación española. 3. Algunas soluciones de derecho comparado. 4. Una efímera atención a un tercer sexo en Francia: la Sentencia del Tribunal de Tours de 20 de agosto de 2015. 5. La Sentencia del TC alemán (BVG) de 8 de noviembre de 2017: reconocer una tercera opción. VI. INTERSEXUALES Y LEGISLACIÓN ANTIDISCRIMINATORIA: 1. La apuesta de la Unión Europea. 2. Australia como avanzadilla: żuna opción a emular? 3. Intersexuales y discapacitados. VII. LAS LEYES AUTONÓMICAS DE TUTELA DE LOS LGTBI Y CONTRA LA DISCRIMINACIÓN VIII. ALGUNAS REFLEXIONES. BIBLIOGRAFIA.

\section{PRESENTACIÓN}

A día de hoy, un segmento significativo de la ciudadanía desconoce qué implica la condición «intersexual $»^{2}$, acaso porque aún es un tema tabú ${ }^{3}$

2 Afortunadamente, en los últimos años algunas películas/novelas han acercado el tema a la sociedad. Así, $X X Y$ [premio Goya a la mejor película extranjera de habla hispana (2007)] o el documental de la BBC, Me, my sex and I. En literatura, la estupenda Middelsex, de Jeffrey Eugenides (ed. Anagrama, 2006).

3 Ghattas (2013: 7). Como señala el $\$ 8$ apdo. LISocial, de la ley 12/2015, de 8 de abril, de Extremadura (véase infra apdo. VII): «Se trata de una realidad escasamente 
que horada un principio que parece incuestionable: la ordenación binaria de los sexos. Se consideran intersexuales las personas que no pueden ser identificadas biológicamente de modo inequívoco como pertenecientes al género femenino o masculino dada la existencia de singularidades cromosómicas, hormonales y/o anatómicas. Así los define el art. 1 de la Ley 2/2016, de 29 de marzo, de identidad y expresión de género e igualdad social y no discriminación de la Comunidad de Madrid: «3. Intersexualidad: Variedad de situaciones en las cuales, una persona nace con una anatomía reproductiva o sexual que no parece encajar en las definiciones típicas de masculino y femenino».

Entre un $0,1 \%$ y un $4 \%$ de la población es intersexual (Scherpe, 2011: 111), aunque el cálculo difiere en función de qué índices se observen y de las concepciones geográficas y culturales (Davidian, 2011: 3-4). Históricamente, la intersexualidad se ha vinculado a la ambigüedad sexual de los genitales - que conlleva centrarse en la denominada cirugía normalizadora-. Ahora bien, es una condición más compleja, con el plus de que no todos los intersexuales se identifican al nacer: en ocasiones, su condición se descubre en la pubertad, al buscar el porqué de la infertilidad o a raíz de controles deportivos (Peterson, 2010: 315-335) ; en algún caso, se conoce en la autopsia.

La invisibilidad del colectivo intersexual está superándose en los últimos años, ante la concienciación de que las — habituales - cirugías de reasignación de sexo suponen una grave lesión de sus derechos fundamentales; en concreto, de su integridad física, de su libertad y de su identidad sexual: desde luego, atentan in genere contra el libre desarrollo de su personalidad. De ahí la atención de las instancias nacionales e internacionales. Así, el informe de la ONU sobre tortura («Report of the Special Rapporteur on torture and other cruel, inhuman or degrading treatment or punishment», del 2013, denunció por primera vez la mutilación genital intersexual e instó a los Estados a derogar cualquier ley que permitiera tratamientos irreversibles. El Consejo de Europa ha mostrado una preocupación insistente, que avala el documento del Comisionado de Derechos Humanos (2015), «Human Rights and Intersex

conocida y tratada en la realidad española». Sin embargo, en los últimos quince años se producido una eclosión de bibliografía jurídica anglosajona sobre la intersexualidad que moldea este trabajo (Newcombe, 2017: 221-267).

4 Fue tristemente célebre el caso de Ma José Martínez Patiño, saltadora de vallas, que no superó el test cromosómico en el Campeonato de España de 1986 (su apariencia - genital también - y sentir eran inequívocamente femeninos, pero tenía síndrome de insensibilidad a los andrógenos). Véase Berghausen (2011: 1188) y Greenberg (1999:273); en general, Glazer (2011-2012: 545-580). 
People» ${ }^{5}$. Su acción clave son las resoluciones 152/2013 y 2191/2017 de la Asamblea Parlamentaria (véase infra apdo. III). La Agencia Europea de Derechos Fundamentales (FRA) publicó en 2015 «The fundamental Rights Situation of Intersex People», un documento — en terminología UE— «ejecutivo» que sintetiza los extremos que les preocupan. Ha de leerse en conjunción con otro informe, "Protection against discrimination on grounds of sexual orientation, gender identity and sex characteristics in the EU. Comparative legal analysis Update 2015» y completarse con el informe "Trans and intersex people. Discrimination on the grounds of sex, gender identity and gender expression» (2011) que encarga la DG de Justicia de la Comisión Europea (Agius y Tobler, 2011) ${ }^{6}$. Todos tienen presentes los «Principios de Yogyakarta sobre la aplicación de la legislación internacional de derechos humanos en relación con la orientación sexual y la identidad de género», un respetado texto no oficial del 2006, ampliado en el $2017^{7}$.

En los últimos años menudean análisis procedentes de organismos rigurosos. En el 2005, se publicó el «Informe de la Comisión de Derechos Humanos de la ciudad de San Francisco", a partir del testimonio de muchos intersexuales. En el 2009, la Human Rights Comission australiana publica "Surgery on intersex infants and human rights», un trabajo pionero. En el 2010, el Comité Nacional de Bioética italiano presentaba el informe «I disturbi della differenziazione sessuale nei minori: aspetti bioetici». Dos años después, la Swis National Advisory Comission on Biomedical Ethics hacía lo propio con una inmejorable exposición — quizás la citada más a menudoEn el 2013, el Deutsche Ethikrat publicó una opinión exhaustiva ${ }^{8}$, que moldea la reforma legal alemana del mismo año. En el 2014, aparece el trabajo

5 En el 2010 ya se había apuntado su situación. Véase Hammarberg (2010).

6 A completar con las «Directrices para promover y proteger el disfrute de todos los derechos humanos por parte de las personas lesbianas, gays, bisexuales, transgénero e intersexuales» del Consejo de la UE, de 24 de junio de 2013 [11153/13 COHOM 125 COPS 240 PESC 728].

7 Del 6 al 9 de noviembre de 2006, especialistas de veinticinco países se reúnen en la Universidad de Gadjah Mada (Yogyakarta) y consensuan los (veintinueve) «Principios sobre la aplicación de la legislación internacional de derechos humanos en relación con la orientación sexual y la identidad de género». Son una declaración sin valor normativo, pero también una guía recurrente, basada en su auctoritas. El 10 de noviembre de2017 se acuerdan en Ginebra los «Yogyakarta Principles plus 10» $(Y P+10)$, que incluyen nueve principios más.

8 También el informe de la Fundación Heinrich Böll (Ghattas, 2013) y los que menciona la Sentencia del BVG de 8 de noviembre de 2017 (véase infra apdo. V.5). 
del Netherlands Institute for Social Research (Lisdonk: 2014) ${ }^{9} \ldots$ Cerramos la relación con el informe de HRW-InterACT, "I Want to Be Like Nature Made Me". Medically Unnecessary Surgeries on Intersex Children in the US».

Como otros materiales valiosos para situar las cuestiones jurídicas que afectan a las personas intersexuales, debemos destacar las resoluciones de los tribunales constitucionales (pensamos en el colombiano y el alemán), las leyes autonómicas y las proposiciones de ley presentadas en el $2017^{10}$ y $2018^{11}$ para reformar o derogar la Ley 3/2007. Por último, las aportaciones de las asociaciones de intersexuales ${ }^{12}$, que constituyen un supuesto emblemático de activismo inteligente.

\section{LA CONDICIÓN DE INTERSEXUAL}

\section{UN CONCEPTO MARCO}

El componente intersexual integra las diferentes alteraciones no encuadrables en la clasificación binaria-omnicomprensiva hombre/mujer (Greenberg, 1999: 278-92 y 2011: 853 $)^{13}$ : el prefijo «inter» identifica todas esas posiciones intermedias (Köllen, 2016: 4) y permite hablar de concepto-marco ${ }^{14}$. Difiere del hermafroditismo, aunque a veces se emplee como un sinónimo (Wacke, 1990: 677-711). En su conceptuación clásica, los hermafroditas incorporan ambos sexos íntegramente - la escultura del hermafrodita Borguèse en el Louvre-; en los intersexuales, la concurrencia de índices de ambos sexos varía en cada caso.

9 El informe del FRA contiene una relación de iniciativas de los países europeos (Austria, Suecia...), aunque convierte la legislación del País Vasco en general (FRA, 2015: 8-10). Proposición de ley para la reforma de la Ley 3/2007, de 15 de marzo, para permitir la rectificación registral de la mención relativa al sexo y nombre de los menores transexuales (BOCG, Congreso de los Diputados, serie B, núm. 91-1, de 3 de marzo de 2017).

11 Proposición de ley sobre la protección jurídica de las personas trans y el derecho a la libre determinación de la identidad sexual y expresión de género (BOCG, Congreso de los Diputados, serie B, núm. 220-1, de 02 de marzo de 2018).

12 ISNA, http://www.isna.org; OII (Organization Intersex International) http://oiiinternational.com; Accord Alliance, http://www.accordalliance.org; Advocates for Informed Choice, https://aiclegal.wordpress.com.

13 Preciosa la presentación del FRA: «Intersex is a collective term for many natural variations in sex characteristics. It is not a medical condition» (FRA, 2015: 2).

14 Por todos, FRA (2015: 2) y Ghattas (2013:10). 


\section{LA DENOMINACIÓN «IDENTIFICATIVA» DE LAS PERSONAS INTERSEXUALES}

La denominación «intersexual» es controvertida. En el ámbito médico, se adoptó en el 2006 el acrónimo DSD (disorders of sexual development), a partir de los debates de la Lawson Wilkins Pediatric Endocrine Society y la European Society for Paediatric Endocrinology que darían como resultado el «Consensus Statement on Management of Intersex Disorders». Para los especialistas, el término intersexual era potencialmente peyorativo (en la biología animal se asocia a organismos primarios) y confundía tanto a profesionales como a progenitores y otros miembros de las familias afectadas (Reis, 2007: $537)^{15}$. Pero para los intersexuales, el término desórdenes evocaba una patología y comportaba que un médico diagnosticase su condición; también promovía cierta exclusión homofóbica (no les convenció que la $\mathrm{D}$ pudiese leerse como differences). Luego, la quinta edición del American Psycologists Association (2013) lo reemplaza por «disforia de género» (pensada para los transexuales, baste recordar la reforma del art. 93 LRC en el 2007), que incluye a los intersexuales, cuya asignación inicial de sexo no se corresponde con su género. Tampoco ha convencido, por reduccionista (Kraus, 2015: 1147-1163) ${ }^{16}$. En suma, el colectivo sigue prefiriendo intersexual, el nomen que avalan los informes internacionales o las leyes consultadas.

\section{SEXO, GÉNERO, ORIENTACIÓN SEXUAL, IDENTIDAD DE GÉNERO}

La condición intersexual deriva de la concurrencia de subfactores ligados al sexo que no se alinean de manera integral con uno de los dos sexos (índices cromosómico, gonadal, morfológico interno y externo, hormonal, fenotípico y — también — sexo de asignación y de crianza e identidad sexual $\left.{ }^{17}\right)$. La falta de un alineamiento «sin fisuras» de los diversos índices comporta un elemento intersexual de mayor o menor envergadura que contesta la idea tradicional de que el sexo, por su condición biológica, es inmutable: mujer u hombre ${ }^{18}$.

15 Hupf (2015: 76-77) procura una crónica de ese rechazo al DSD.

16 En el 2018 está previsto revisar categorías y podría afectar a este criterio.

17 Por todos, Greenberg (1999: 278) y García López (2015: 57-59).

18 Como última aproximación, la EM Ley foral, 12/2009: « [...] el sexo va más allá de la simple apreciación visual de los órganos genitales externos presentes en el momento del nacimiento: como ha ido estableciendo la ciencia médica moderna, se trata de una realidad compleja, consecuencia de una cadena de eventos cromosómicos, gonadales y hormonales, entre otros, que en su sucesión determinan lo que comúnmente conocemos como hombres y mujeres; dicha cadena de eventos sufre en ocasiones rupturas 
En las construcciones actuales, el concepto de "sexo" se opone al de "género», empleado para identificar las cualidades culturales y de actitud características de un determinado sexo (Greenberg, 2002: 149-159). Parecería, pues, que están en planos distintos, pero la realidad es más compleja. Hoy ya no hablamos de sexo en términos únicamente biológicos, sino performativos (Hester, 2004: 28) y con un componente social ${ }^{19}$. El sexo ya no es concebible como una categoría fija y eso supone contestar la férrea distinción binaria. Aunque intentemos deslindarlos a efectos jurídicos para situar, por ejemplo, las herramientas técnicas contra la discriminación, acabamos aceptando parcelas difusas, incluso una cierta fungibilidad terminológica.

Curiosamente, la distinción entre sexo y género se asienta en el tristemente célebre caso de David Reimer (conocido como John/Joan), un caso que se vincula a la intersexualidad, aunque en puridad no lo sea. Con ocho meses, David perdió el pene en una circuncisión. Sus padres acudieron al prestigioso psicólogo John Money, quien sostenía que los niños eran psicosexualmente neutrales al nacer, por lo que podían integrarse en cualquier género una vez se adecuase su anatomía sexual (una apariencia «normal» de sus genitales era fundamental). La adecuación, para garantizar el éxito, debía ser antes de los dos años, y luego centrarse en una meticulosa crianza de acuerdo con el género asignado - el Optimal Gender Rearing, en denominación al uso-. David fue criado como una niña, sufrió varias operaciones para reconstruir unos genitales ad hoc y fue objeto de seguimiento médico durante su infancia ${ }^{20}$, como garantía de esa feliz adscripción al género femenino publicitada en importantes revistas científicas. A día de hoy, la teoría de Money todavía justifica en parte la política de la Academia Americana de Pediatría. Se ignora que David rechazó muy pronto el género femenino, que en la adolescencia recuperó el masculino, que confesó en un libro el calvario padecido y que se suicidó a los 38 años. La proclamada flexibilidad del género se revelaba, en su caso más célebre, una impostura.

y diferenciaciones que producen como resultado la existencia de personas con características cruzadas de uno y otro sexo» (sobre la ley, véase infra apdo. VII).

19 "Traditionally, a distinction is drawn on the basis that sex is seen as a fact, defined by biology, while the definition of gender is constructed by the culture in which an individual lives. However, the distinction between sex and gender is not as straightforward as this [...] the "biological fact" of sex is only a "fact" of any interest because of the cultural importance attached to it [...]. Further, gender (the roles expected of men and women) may in part reflect the assumed differences of their bodies» (Chau y Herring, 2002: 328).

20 Sobre la historia, White (2014: 784-788); Hermer (2002: 202 ss); Davidian (2011: 4-5) o Uslan (2010: 302-303). 
Pese a lo anterior, los conceptos de sexo y género sitúan hasta la fecha las categorías técnicas de discriminación por razón de sexo, orientación sexual e identidad de género ${ }^{21}$. Y surge una cuestión: ¿cuál es la ubicación sistemática de los intersexuales? En el informe «Derechos humanos e identidad de género» del comisario de Derechos Humanos del Consejo de Europa (2010) ${ }^{22}$ se concatena intersexuales e identidad de género:

La identidad de género es uno de los aspectos más fundamentales de la vida. Habitualmente, se asigna el sexo de una persona al nacer, convirtiéndose a partir de este momento en un hecho social y legal. Sin embargo, un número relativamente pequeño de individuos tienen problemas con pertenecer al sexo registrado al nacer. Lo mismo puede ocurrir con personas intersexuales cuyos cuerpos, y en ocasiones su anatomía genital, incorporan ambos o ciertos aspectos tanto de la fisiología masculina como femenina. Para otras personas, los problemas surgen porque su autopercepción innata no está en conformidad con el sexo que se les asignó al nacer. A estas personas se les hace referencia como personas «transgénero» $\mathrm{O}$ «transexuales».

Esta afirmación demuestra el uso — parcialmente- fungible que apuntábamos. En puridad, la intersexualidad debe vincularse al índice «sexo». Lo recuerda la FRA: «It is also important to bear in mind that intersex refers to the bodily characteristics of a person. There is no evidence linking specific sex characteristics with either gender identity or sexual orientation. Thus, intersex people might be as likely as non-intersex people to self-identify as heterosexual, bisexual, homosexual, trans, etc. ${ }^{23}$.

21 Por todos, Skougard (2011: 1165).

22 «Algunos marcos legales de los estados miembros del Consejo de Europa categorizan, desafortunadamente, la identidad de género bajo el término de "orientación sexual", lo que no es exacto ya que la identidad de género y la orientación sexual son dos conceptos diferentes. La orientación sexual debería ser entendida como la capacidad de cada persona para una atracción emocional, afectiva y sexual hacia, y para las relaciones íntimas y sexuales con individuos de diferente o igual género, o de más de un género (heterosexualidad, homosexualidad y bisexualidad)» (Hammarberg, 2010: 4).

23 FRA (2015: 4) Y concluye: "While there may be some overlap between the grounds of "sex", "gender identity" and "intersex status", it is important that intersex status is protected as a separate ground because people who are intersex are also vulnerable to discrimination. It also recognizes that discrimination on this ground manifests differently to discrimination on the grounds of sex and gender identity». 


\section{LOS INTERSEXUALES COMO INTEGRANTES DE LA SECUENCIA LGBT: AHORA LGBTI}

En la asamblea del 2009, la Asociación LGBT —lesbianas, gays, bisexuales y transexuales - aceptó incorporar a los intersexuales, adoptándose la secuencia LGBTI. No todos los intersexuales estuvieron conformes. Temían diluirse si se concatenaban a otros grupos con problemáticas específicas.

Ciertamente, a partir de los índices mencionados anteriormente, podemos diferenciar sin problemas intersexuales y homosexuales. Los segundos no se ligan al sexo sino a la orientación sexual, lo que no obsta para que puedan existir intersexuales con diferente orientación sexual (lo veíamos antes en el informe del FRA). La distinción resulta más compleja respecto de los transexuales. ${ }^{24}$. Frente a los intersexuales, los transexuales experimentan una clara discrepancia entre el sexo con el que nacen y su sentimiento de pertenencia al otro. Esa distinción tiene — no obstante- fisuras, dado que personas intersexuales asignadas a un sexo al nacer, desde la premura o la arbitrariedad de la asignación, pueden sentir una identidad sexual que difiere de la legal y que no nace - como en el caso de los transexualesdel cambio, en la ordenación binaria, de un sexo «único» hombre/mujer al otro, sino de la inexacta identificación inicial, que el crecimiento del sujeto contesta $^{25}$. Son, se dice, un $25 \%$ del colectivo (Vanderhorst, 2015: 265). Además, la irrupción del concepto "transgénero" ${ }^{26}$ impone nuevas reflexiones. El término «trans» designa un abanico de supuestos mucho más amplio que el de transexual ${ }^{27}$, pues tienen cabida agéneros, bigéneros, andróginos, cross-dresser, o genderqueer. En esa secuencia a menudo se incluye a los intersexuales, pero no es exacto y tampoco una opción generalizada (basta

24 "Intersex and transgender individuals can face common issues concerning their gender and sex, but the terms "intersex" and "transgender" are not interchangeable. While the term "intersex" refers to a biological phenomenon, "transgender" encompasses a spectrum of individuals' self-identifications and gender expressions that do not match one's assigned sex" (White, 2014: 779).

25 Como espetó Anne Tamar-Mattis, respetada activista interesexual, «if the child later decides that the guess [of the doctor] was wrong, is that a sign that something is wrong with the child?» (Agius, 2015: 23).

26 Que encontramos en algunas leyes, como la catalana, extremeña, madrileña o balear (véase infra apdo. VII).

27 El asterisco como parte de la palabra: «The asterisk actually pushes beyond the trans-prefix and opposes it as the only legitimate way to refer to trans* identities and communities» (Tompkins, 2014: 27-28). 
recordar la rúbrica de algunas leyes autonómicas $\left.{ }^{28}\right)$. A día de hoy coexisten esfuerzos por encontrar similitudes y/o diferencias, e incluso se da una reciprocidad peculiar en el caso de transexuales que se arrogan la condición intersexual ${ }^{29}$. Los une, pese a todo, una reivindicación primaria: la necesidad de superar la mirada médica que los califica de desórdenes ${ }^{30}$. De hecho, la acepción "trans» se define por la superación de tratamientos médicos y acciones sobre el cuerpo, para centrarse en la voluntad, incluso si temporal o itinerante, de los sujetos ${ }^{31}$. Y en esa superficie, tan difusa, caben — quizás a su pesar- los intersexuales como realidades que exceden el colectivo «cisexual». En definitiva, cabe defender un tratamiento conjunto a partir de la constatación de la diferencia ${ }^{32}$.

A los efectos de este trabajo importa retener el nexo entre intersexuales y LGTB en el diseño de las políticas públicas y contra la discriminación. Quizás porque la secuencia, en acertada observación de Benavente Moreda (2013b: 120), los muestra como realidades que merecen un tratamiento diferente pero partiendo de que "todas ellas reflejan "en positivo" una forma de manifestar el derecho de la persona a su propia identidad». Aquí emerge, además, el concepto de identidad sexual, que algunos análisis estiman sinónimo de identidad de género, pero que también puede entenderse como macroconcepto que incorpora los índices, sexo, orientación sexual, identidad de género y expresión de género. Es probablemente la acepción que se consolidará en los próximos años.

28 Véase infra apdo. VII. Un estupendo glosario de neoterminologías en Agius y Tobler (2011: 88-90).

29 «Admittedly, however, there are also people (classified as transsexual) who describe themselves as intersexed although not diagnosed with DSD, attributing this to the hormonal situation of their body, and specifically of their brain» (Deutsche Ethikrat, 2013: 25).

30 "Both groups, while part of the normal spectrum of anatomical and psychological variation, are classified by society as "disordered"; transgender individuals have "disordered minds", intersex people have "disordered bodies", and both must be "fixed" lest they undermine society's rigid division of male and female» (Vanderhorst, 2015: 266).

31 Como se apunta en el IV\$2 EM Prop. Ley (2018), todo se fundamenta en «el derecho a la libre determinación de la identidad sexual y expresión de género, que es la capacidad que tienen las personas para autodefinirse, incluyendo la configuración y gestión de su propio cuerpo".

32 El management que desarrolla Köllen (2016: 1-20). 


\section{CLAVES PARA EL ANÁLISIS JURÍDICO: LAS RESOLUCIONES DE LA ASAMBLEA PARLAMENTARIA DEL CONSEJO DE EUROPA}

Los pronunciamientos más útiles para situar los desafíos jurídicos de la intersexualidad corresponden, creemos, a la Asamblea Parlamentaria del Consejo de Europa. En primer lugar, la Resolución de 152/2013 sobre «children's right to physical integrity", que visibilizaba la preocupación ante diferentes lesiones de la integridad física de los menores, mayoritariamente asociadas a supuestos que sus valedores — las familias... - han defendido histórica y culturalmente como beneficiosos para ellos, «despite clear evidence to the contrary». Dicha resolución suscitó numerosas críticas porque concatenaba mutilación genital femenina, circuncisión por razones religiosas, intervenciones médicas tempranas en caso de niños intersexuales y sumisión de los niños en relación con piercings, tatuajes u operaciones estéticas. En relación con los intersexuales, proponía garantizar que nadie padeciese operaciones quirúrgicas innecesarias durante su infancia y juventud y por ende la integridad física y la autonomía decisoria de los afectados.

Pero la resolución más importante es la 1291/2017: «Promoting the human rights of and eliminating discrimination against intersex people», que tiene - por fin - una visión global y que en relación con las operaciones establece:

7.1.1. Prohibit medically unnecessary sex-"normalising" surgery, sterilisation and other treatments practised on intersex children without their informed consent;

7.1.2. ensure that, except in cases where the life of the child is at immediate risk, any treatment that seeks to alter the sex characteristics of the child, including their gonads, genitals or internal sex organs, is deferred until such time as the child is able to participate in the decision, based on the right to self-determination and on the principle of free and informed consent [...].

También atiende a los aspectos de la inscripción registral (la otra cuestión nuclear), y explicita — relevante— la posibilidad de fijar mecanismos de compensación económica ${ }^{33}$.

33 «7.5.1. [...] conduct an inquiry into the harm caused by past invasive and/or irreversible sex-"normalising" treatments practiced on individuals without their consent and consider granting compensation, possibly through a specific fund, to individuals having suffered as a result of such treatment carried out on them». 


\section{LAS OPERACIONES «CORRECTIVAS» TEMPRANAS}

\section{LA OPERACIÓN COMO EMERGENCIA SOCIAL: ACTUAR «POR EL BIEN DE TODOS»}

La atención a las personas intersexuales se ha centrado en las cirugías de adaptación —o de "normalización»— tempranas, en casos de órganos genitales ambiguos ${ }^{34}$. Durante las últimas décadas, identificada la situación, el cuadro médico comunicaba a los progenitores la necesidad de operar, para reconducir a los recién nacidos a las categorías conocidas ${ }^{35}$ y poder inscribirlos en el registro.

Habitualmente la operación era el punto de partida de un calvario clínico: durante la infancia se sucedían otras operaciones ${ }^{36}$, tratamientos hormonales, revisiones periódicas y exposiciones clínicas - como si el menor fuera un espécimen de feria—. La supervisión parental incluía impedir manifestaciones que contestasen las convenciones del género asignado (el niño que se disfraza de hada). Por último, los protocolos fijaban un secretismo absoluto en interés del menor. El fundamento, siempre, el caso de John/Joan.

A partir de los ochenta del siglo pasado fue evidente que las operaciones tempranas conllevaban disfunciones en el organismo de los intersexuales adultos (dolores musculares, problemas endocrinos...) y perjuicios psicológicos severos (sentimiento de vergüenza, depresión, etc.) y comenzaron a cuestionarse.

A estas alturas, las denuncias son conocidas, pero, pese a ello, se da una "persistence of medically unnecessary surgery» que constata en el 2017 el informe de HRW-InterACT (2017: 47-52). La FRA afirmaba en el 2015 que al menos en veintiún Estados europeos miembros continuaban realizándose operaciones «normalizadoras» (FRA, 2015: 1). Se apunta como razón última que los cuadros médicos todavía están convencidos de la probidad de dichas operaciones: un niño sin sexo no es un ser humano ${ }^{37}$. Consideran —aunque cada vez discrepen más—

34 Aunque hay muchos otros supuestos, como la remoción intempestiva de órganos internos que no se ajustan con los externos...

35 La mayoría de niños «dudosos» se reconducen al género femenino: resulta más sencillo reconstruir una vagina virtual que un pene standard. Véase Ford (2001: 471).

36 "Simply, it is difficult, if not impossible, to construct genitals on a one-year-old child that will look and function well when the child begins puberty and again when the child enters adulthood» (Puluka, 2015: 2130).

37 "The surgery is a gift of a rights-bearing body and an existence as a legal subject and a citizen» (Bird, 2005: 66). "Doctors perform cosmetic genital surgery on infants with an intersex condition because they believe that people with a DSD will suffer psychological harm as children and as adults. They think that children who grow up with genitalia 
beneficioso operar desde una triple perspectiva: 1) hace efectivo el mejor interés del menor, pues lo «normaliza» de acuerdo con los parámetros existentes ${ }^{38} ; 2$ ) preserva los valores sociales de la ordenación binaria (Hester, 2004: 32-34) ${ }^{39}$; 3) salvaguarda la salud de los progenitores, evitándoles enfrentarse a la «anormalidad» de su hijo en cada cambio de pañales (Lloyd, 2005: 173).

En esa argumentación hay, no obstante, una falla: en la mayoría de los casos, la operación no es necesaria desde una perspectiva médica ${ }^{40}$. Muy frecuentemente se califica de mera operación "cosmética» (Kismödi y Shakespeare, 2014: 2). Pero en el criterio médico - la American Pediatric Association (Puluka, 2015: 2133) — la ambigüedad sexual se convierte en emergencia «social» ${ }^{41}$, con el agravante de la exigencia de una pronta inscripción en los registros civiles (fijar un «sexo legal»). La noción de emergencia (social) permite operar sin mayores disquisiciones e, incluso, excluir el consentimiento ${ }^{42}$

that look different from their peers' genitalia will suffer emotional trauma. They also believe that adults with atypical genitalia will face obstacles in forming romantic relationships or engaging in reproductive or other sexual acts» (Greenberg, 2011: 897). "The theory supporting early surgical intervention is that a child who has normative-looking genitals from a very early age, and is raised "unambiguously" in the gender that matches those genitals, will develop the desired gender identity regardless of chromosome pattern, body structure at birth, or hormone exposure in the womb. Many practitioners also believe that parents will be unable to accept or bond with their intersex children without genital-normalizing surgery, and that children with atypical genitals will suffer teasing from peers and rejection from potential partners as they grow» (Tamar-Mattis, 2006: 64).

Puntualiza Puluka: «Surgery often serves more of a social than a medical Purpose, allowing doctors, parents and society to avoid the discomfort of conceptualizing gender, outside of the strict gender binary» (Puluka, 2015: 2128).

40 "The intersexed infant is in no immediate danger because of the size and shape of his/ her penis or clitoris. In fact, the intersexed infant is not even in future danger because of the size and shape of his/her penis or clitoris» (Ford, 2001: 476).

41 «Interestingly, even though the medical establishment has admitted that the vast majority of intersex births pose no "medical" emergency requiring treatment, it maintains that the condition of intersexuality is a medical issue, substituting a rubric of "social" emergency and psychological health to justify physical intervention as close to birth as possible» (Reilly, 2005: 303). Asimismo, Benson (2005-2006: 35).

42 En el asunto Schloendorff v. Society of New York Hospital (1914), el juez Cardozo determinó que la existencia de emergencia excluía el requisito del consentimiento informado. «It is this determination that may underlie the centrality and persistence of the clinical characterization of intersex as an emergency that the existence of an emergency negated the requirement of informed consent» (Holmes, 2005: 129); también Ford (2001: 475). 
aunque en la práctica los médicos lo obtienen con facilidad de unos padres ansiosos por procurar la «normalidad» para su hijo ${ }^{43}$.

La situación está cambiando. En el 2006 se publicó el «Consensus Statement on Management of Intersex Disorders», que ya no consideraba la operación como la solución idónea en todos los supuestos (la atención al sexo cromosómico modula el criterio monolítico: la genitalidad externa ya no es suficiente). Paralelamente, se ha constatado que los menores intersexuales no operados tienen un desarrollo psicosocial sano ${ }^{44}$ y que un número muy significativo de intersexuales adultos operados tempranamente hubieran preferido que la intervención no se produjera ${ }^{45}$. Por último, el número de organismos internacionales que propugnan evitar intervenciones innecesarias crece a diario, aunque - no nos engañemos-, en ese debate externo al ámbito sanitario, también hay defensores de las cirugías en beneficio de los menores ${ }^{46}$.

Todo ello conduce a dos posiciones bien definidas: por un lado, quienes propugnan una moratoria sin matices de cualquier operación durante la minoría de edad, para garantizar que sea el intersexual quien decida y porque los progenitores, en el ejercicio de sus funciones parentales, no pueden tomar decisiones que por su magnitud afectarán toda la vida adulta de sus hijos ${ }^{47}$ (¿cómo van a privar a los menores intersexuales de escoger libremente su género cuando crezcan ${ }^{48}{ }^{4}$ ). Por el otro, se mantiene la opción de la operación, siempre que se fundamente en un consentimiento informado adecuado (Benson, 2005: 37 $)^{49}$. Y se enfatiza la singularidad de cada supuesto.

Es importante incidir en que los partidarios de demorar las intervenciones no pretenden criar menores «agénero». Defienden que los progenitores

43 De hecho, durante el siglo pasado algunos médicos sostenían que los progenitores opuestos a la cirugía ejercitaban de modo negligente sus responsabilidades parentales (Morland, 2014: 112).

44 Bishop (2007: 541); Chau y Herring (2002: 353); Deutsche Ethikrat (2013: 143).

45 "The strongest argument against genital-normalizing surgery on infants is that every intersex person who has spoken publicly on the subject has spoken against surgery» (Tamar-Mattis, 2006: 68); véase también Hermer (2007: 255-272).

46 Aliabadi, tras un minucioso análisis, recuerda respecto de la moratoria: «This avenue may be in the best interest of some intersexed infants, but there is no indications that it is in the best interest of all of them» (Aliabadi, 2005: 457).

47 Por todos, Uslan (2010: 308-310). Dedica especial atención al matrimonio.

48 "Given that a parent is completely ignorant to their child's gender identity at birth, it is not appropriate to ask them to make determinations to assign their child a sex, given the complex relationship between sex and gender» (Thorn, 2014: 613).

49 Otra crítica habitual: una vez que los médicos consiguen el consentimiento paterno, se desentienden de otras posibles opciones (Lareau, 2003: 130). 
asignen un género al menor a partir de la información médica recibida. Dicha información les ha de permitir anticipar, con una cierta previsibilidad, cuál será el que él adopte en la pubertad (Bishop, 2007: 544). Es entonces cuando un menor decidirá en su mejor interés, posiblemente manteniendo al género asignado, pero sin lastres impuestos.

\section{LOS DERECHOS FUNDAMENTALES QUE LAS OPERACIONES LESIONAN}

La Resolución 1291/2017 identificaba los derechos del CEDH vulnerados: los arts. 3 [prohibición de tortura] y 8 [derecho al respeto a la vida privada y familiar]. En el art. $8 \mathrm{CEDH}$, justamente, se incluye el derecho a la integridad física y psicológica en los casos en que no se contempla la lesión del art. 3. Pese a que el art. $8 \mathrm{CEDH}$ no explicita un derecho a la autodeterminación del sujeto, la noción de autonomía personal subyace en su interpretación y en la configuración de sus garantías (Agius, 2015: 31 y 32). Asimismo, se vulnera el derecho a la salud como derecho independiente ${ }^{50}$, y específicamente los derechos reproductivos de los intersexuales, pues numerosas operaciones alteran su capacidad reproductiva futura, como recordaba la OMS en el $2014^{51}$. En nuestro país, por tanto acudimos a los arts. 15 y 43. También al art. 10 CE: la dignidad como fundamento de la condición humana.

Desde otra perspectiva conexa, las operaciones suponen una agresión a los derechos de los niños, dado que la inmensa mayoría de las veces tienen lugar durante la infancia y la adolescencia. En concreto, contravienen los arts. 3, 7, 8. 12 y 13 CDN [1989] (Agius, 2015: 33). Y como principio global, atentan contra el libre desarrollo de su personalidad, si sustraen a los menores de intervenir en un aspecto fundamental para su evolución: la fijación de su identidad sexual. Evidentemente, van contra su interés superior ${ }^{52}$. De nuevo,

50 Una acotación conceptual de los derechos a la vida, a la integridad corporal y a la salud, en Asensio (2012: 49-56).

51 «Intersex persons, in particular, have been subjected to cosmetic and other non medically necessary surgery in infancy, leading to sterility, without informed consent of either the person in question or their parents or guardians. Such practices have also been recognized as human rights violations by international human rights bodies and national courts» (Kismödi y Shakespeare, 2014: 2).

52 «Es precisamente el libre desarrollo de la personalidad y los principios constitucionales de protección de los menores y de los hijos del art. 39.3 y 5 CE lo que nos permite interpretar el interés del menor como referido principalmente a la protección de sus derechos y libertades fundamentales, y dado que la finalidad última de la protección es garantizar el libre desarrollo de su personalidad, aparecerán, por esta vía, identificados interés del menor y libre desarrollo de la personalidad» (Asensio, 2013: 
el art. 10 CE, pero también, en su detallada redacción, el art. 2 LOPM que - tras la reforma por LO 8/2015 - recuerda que para identificar ese interés superior del menor, se tendrán en cuenta: «d) La preservación de la identidad, cultura, religión, convicciones, orientación e identidad sexual o idioma del menor, así como la no discriminación del mismo por éstas o cualesquiera otras condiciones, incluida la discapacidad, garantizando el desarrollo armónico de su personalidad».

\section{3. ¿̇QUÉ CONSENTIMIENTO Y QUÉ EMISOR EN CASO DE OPTAR POR LA OPERACIÓN QUIRÚRGICA?}

¿En qué consistirá ese consentimiento informado? ¿Quién debe emitirlo? ¿Es conveniente, dada la magnitud del acto, incorporar algún tipo de asistencia o cortapisa? Cabría pensar que la discusión decae ante la prohibición que ya prescriben algunas leyes (véase infra apdo. VII), pero como todavía es una realidad no residual proceden algunas reflexiones.

Partimos, necesariamente, del concepto de consentimiento informado del art. 3 de la Ley 41/2002, de 14 de noviembre, básica reguladora de la autonomía del paciente y de derechos y obligaciones en materia de información y documentación clínica: «[...] la conformidad libre, voluntaria y consciente de un paciente, manifestada en el pleno uso de sus facultades después de recibir la información adecuada, para que tenga lugar una actuación que afecta a su salud $»^{53}$. Nos conduce a dos datos relevantes: libertad de emisión e información «adecuada», que trataremos después.

\section{1. Materiales para encarar el problema: las decisiones del TC de Colombia}

En Colombia, las tempranas sentencias de 1995 y 1999 (las resoluciones $\mathrm{YY}, \mathrm{XX}$ y $\mathrm{NN}^{54}$ ) sientan de manera pionera criterios para abordar estas operaciones. El fallo emblemático es la Sentencia SU-337/99, respecto de una niña con seudohermafroditismo masculino. Para los médicos, la operación podía ser conveniente pero debía ser la menor quien decidiese cuando tuviera edad

45). Esa conexión entre desarrollo e interés superior del menor es clave para la mejor inteligencia de este tema. Identifica la necesaria búsqueda de la autonomía del menor. Véase al respecto Eekelaar (2006: 156-158).

53 Sobre el consentimiento informado, in genere, Agón (2017), Reynal (2017), Sardinero García (2017) y Asensio (2012: 56-77; en relación con los menores, 77-128).

54 Disponibles en: http://www.corteconstitucional.gov.co. Véase González Sánchez et al. (2010: 204-211); asimismo, White (2014: 795-802). 
para ello. La madre (el padre había fallecido) deseaba una pronta cirugía para evitar perjuicios en el desarrollo físico y psicológico de su hija.

El tribunal fue claro. El art. 16 de la Constitución colombiana establece el libre desarrollo de la personalidad, que incluye el sentimiento de pertenecer a un sexo determinado. De ahí que sea preciso, en estos casos, que el menor preste su consentimiento informado. Ciertamente, los padres podrían sustituirlo con su propio consentimiento informado en su primera etapa, pero la necesidad de dicho consentimiento se diluiría con la edad, ante un menor progresivamente consciente de su identidad de género. Y fijó el límite en los 5 años:

Una obvia pregunta surge: ‘̇a qué edad se puede presumir que han ocurrido los cambios sicológicos que invalidan el consentimiento sustituto paterno en caso de ambigüedad genital de la menor XX? No existe una respuesta clara a ese interrogante, por cuanto las diferentes personas se desarrollan en distinta forma, y existen a veces agudas controversias entre las diversas escuelas psicológicas sobre la manera como los seres humanos evolucionan, desde el nacimiento hasta la madurez [...]. Sin embargo, es importante resaltar que numerosos estudios de psicología evolutiva y las diversas escuelas sicológicas, a pesar de sus obvias diferencias de enfoque, coinciden en general en indicar que a los cinco años un menor no sólo ha desarrollado una identidad de género definida sino que, además, tiene conciencia de lo que sucede con su cuerpo y posee una autonomía suficiente para manifestar distintos papeles de género y expresar sus deseos.

En la Decisión T-551/99 también es relevante la edad de la menor, con hiperplasia suprarrenal, cuya operación se solicita. El endocrinólogo y el cirujano que trataron a la niña prescribieron una operación de remodelación genital a los dos años. Sin embargo, al alcanzar dicha edad, el organismo administrativo no lo autorizó y el progenitor lo solicitó recordando, entre otros datos, la difícil situación que vivían, con la niña preguntando sobre la apariencia inusual de sus órganos genitales. El tribunal se cuestionó si era legítimo el consentimiento sustitutivo paterno y afirmó que solo si era «cualificado y persistente [...] pues esa categoría conceptual constituye el principio normativo general sin el cual resulta imposible decidir en concreto»:

[...] el permiso paterno sustituto es válido para autorizar una remodelación genital en menores de cinco años, siempre y cuando se trate de un consentimiento informado cualificado y persistente, lo cual supone que la comunidad médica debe desarrollar protocolos que permitan cualificar el consentimiento paterno. Igualmente, esta Corporación precisó que a ella no le corresponde elaborar en detalle las reglas precisas que deben contener estos protocolos, pues esa tarea debe ser desarrollada directamente por la comunidad médica, obviamente dentro del marco normativo que fije el Congreso, puesto que, en desarrollo del principio democrático y de la 
cláusula general de competencia (CP arts.1º, $3^{\circ}$ y 150), corresponde al Legislador regular temas de esta naturaleza.

En definitiva, para el TC colombiano no cabe consentimiento sustitutorio desde que el menor cumple cinco años y en todo caso el consentimiento de los progenitores, y del menor cuando proceda, ha de ser "cualificado y permanente», asistidos por equipos pluridisciplinares que acompañen después a las familias en su compleja cotidianidad ${ }^{55}$.

\subsection{La intervención de los progenitores}

Sabemos que los progenitores actúan como representantes legales de sus hijos, excepto en relación con los actos relativos a los derechos de la personalidad u otros que el hijo, de acuerdo con sus condiciones de madurez, puede realizar por sí solo (art. 162.1 CC). Respecto del consentimiento paterno en las operaciones, no cabe hablar de representación stricto sensu: una intervención sustitutoria de los progenitores en relación con los derechos de la personalidad, deberá fundamentarse en el mejor ejercicio de las responsabilidades parentales, y en el deber de velar por sus hijos ${ }^{56}$ (art. 154.3.1 ${ }^{\circ} \mathrm{CC}$ ). Y no será necesaria si estimamos que el menor, para ese supuesto o intervención tiene capacidad suficiente para consentir: lo concreta la normativa sanitaria, pero en última instancia se apoya en el Código Civil.

En caso de operaciones u otros tratamientos irreversibles, el velar por el interés de sus hijos tiene dos implicaciones concretas. 1) El art. 6 del Convenio de Oviedo ${ }^{57}$ establece que la intervención de una persona incapaz de consentir solo puede efectuarse cuando redunde en su beneficio directo (luego atiende al juego y correlaciones de consentimientos). Ese «solo si redunda en su beneficio directo» (aquí el interés superior del menor) cuestionaría que tengan cabida operaciones consideradas, en puridad, cosméticas. 2) Los progenitores han de tener presente que las operaciones tienen consecuencias a largo plazo, no suponen una acción de envergadura pero puntual que permite borrar el tema.

Incluso si consideramos que, en el caso concreto, los progenitores deben ser quienes consientan la intervención (ex art. 9 de la Ley 41/2002), se han

55 El tribunal, pese a esa doctrina, en el caso concreto no autorizó de manera urgente la operación, sino que optó por continuar el tratamiento médico, con la medicación necesaria, etc.

56 Véase Asensio (2012: 36) y Agón (2017:165-166).

57 Convenio para la protección de los derechos humanos y la dignidad del ser humano con respecto a las aplicaciones de la Biología y la Medicina (BOE núm. 252, de 20.10.1999 —instrumento de ratificación-) 
detectado una serie de disfunciones en la formación libre e informada de su consentimiento:

1) La capacidad racional de los progenitores en el momento de consentir la operación está afectada por un coctel de sentimientos de desesperación, tristeza, incluso horror. Aunque creerán actuar en interés del menor, están sometidos a una presión inimaginable durante el embarazo. De hecho, se ha justificado la operación como instrumento para consolidar los vínculos entre progenitores e hijos "no normales» y evitar el rechazo ${ }^{58}$.

2) ¿En qué debe consistir la información en este caso? Sabemos que la información ha de tener un contenido mínimo (art. 4 de la Ley 41/2002), que incluye la naturaleza de la intervención, su finalidad, sus riesgos y sus consecuencias. Ex art. 10.1, se identifican las consecuencias relevantes o de importancia que la intervención origina con seguridad; los riesgos relacionados con las circunstancias personales o profesionales del paciente, los riesgos probables en condiciones normales, conforme a la experiencia y al estado de la ciencia o directamente relacionados con el tipo de intervención y las contraindicaciones (Reynal, 2017: 70-80). Pues bien, parece que en muchos supuestos los progenitores reciben una información parcial y «tendenciosa». Como los médicos no contemplan la posibilidad de posponer la operación, no presentan alternativas viables ${ }^{59}$. Para conseguir el consentimiento "cualificado y permanente» que exigían las sentencias del TC colombiano, parece conveniente la asistencia de un equipo pluridisciplinar que contribuya a la mejor conformación de la voluntad de los progenitores - y como

58 La operación «relieves parental distress and improves attachment between the child and the parents» (Davidian, 2011: 17). «Occasionally caregivers might be so upset about their children's uncertain gender situation, so scared about how their children might develop, so overwhelmed by the issue of identity, that they are unable to maintain an aceptable and close relationship" (Miano, 2015: 594).

«Doctors, perhaps acting out of concern for the child's well-being or the parents' comfort, frequently filter information in such a way that parents make the, decision to authorize genital-normalizing surgery on the basis of incomplete information and without having considered other options» (Tamar-Mattis, 2006: 86). Añade que los padres que reciben únicamente información médica son el triple de numerosos en consentir la operación que quienes reciben una información transversal, con intervención de psicólogos — lo asume Agius (2015: 23) —, aunque se cuestiona (Muckle, 2006: 1007). Estremecedor el título —y el contenido, en que se deconstruye la narrativa médica — del artículo de Hester (2004: 21-49): «Intersex(es) and informed consent: how physician's rhetoric constraints choice». 
antídoto, incluso a aventuras quirúrgicas ${ }^{60}$ - Así lo afirman reiteradamente expertos de muy diversa procedencia. E incluso se plantean otras fórmulas: algunas organizaciones intersexuales proponen que los progenitores, para encarar la situación, hablen con adultos que nacieron con componente intersexual (Puluka, 2015: 2314) ${ }^{61}$.

\subsection{La asistencia de terceros}

Los equipos que mencionábamos facilitan que el consentimiento parental se forme adecuadamente. Pero surge una alternativa, distinta, que también se baraja. Si para determinados actos, por su relevancia (por ejemplo, patrimoniales) es necesaria la autorización del juez, ¿por qué dejar a los progenitores solos en esta materia? ¿No sería más beneficioso idear mecanismos que permitieran compartir la responsabilidad?

En este supuesto, entre los progenitores y los menores, incluso si los confrontamos (dado que se admite que los padres tienen intereses propios...) no cabe hablar de conflicto de intereses stricto sensu, porque el interés superior del menor prevalece en cualquier situación que le afecte (art. 2 LOPJM). Eso no obsta para que, al imaginar algún tipo de intervención "colaboradora» de un tercero se lo llegue a tipificar como «defensor judicial del menor» (Curtis, 2011: 845) —técnicamente discutible- Esa parece ser la opción de Italia y Holanda, en tanto que en otros, como Lituania, el tercero dirimente es el propio cuadro médico ${ }^{62}$. En Malta —véase infra —, la legislación específica sobre intersexuales contempla una comisión nombrada por el ministro (renovable cada tres años). Otra opción sería la intervención de los tribunales, aunque no estemos ante uno de los actos sujetos stricto sensu a aprobación judicial (art. 166 CC). Quizás fuera óptimo regular una intervención ágil del Ministerio Fiscal, convenientemente asesorado.

\subsection{La participación (consentimiento) del menor}

El art. 9 de la Ley 41/2002, tras la reforma de la Ley 26/2015, de 28 de julio, de modificación del sistema de protección a la infancia y a la adolescencia

60 «Some researchers have found that while surgery makes parents feel more comfortable, counseling can serve the same purpose without the disadvantages of irreversible surgery» (Lareau, 2003: 136).

61 La OMS proponía: «Provide support and information to parents who have intersex children, for example through psychological counselling and peer support or self-help groups» (Kismödi y Shakespeare, 2014: 15).

62 FRA (2015: 7). En una primera lectura, que decida el cuadro médico perpetúa el problema. 
(DF 2a), refuerza la protección del menor respecto del consentimiento informado $^{63}$. Se mantiene la figura del menor maduro, pero — si se nos permite la licencia - en cierta medida "atornillado» para los casos de gravedad ${ }^{64}$ (art. 9.4.3).

En este supuesto no estamos ante una situación de grave riesgo, aunque sí ante una operación que va a determinar la evolución y desarrollo del menor. No se trata de una persona enferma, pese a las inercias sanitarias, sino de un supuesto a revisar en términos de autonomía y bienestar físico y psíquico del concernido (el menor intersexual). Por eso, la atención a su madurez, a su libre discernimiento, ha de ser el parámetro determinante.

Habida cuenta de esa "contigüidad» — práctica, que no conceptualentre transexuales e intersexuales, cabría atender a los análisis sobre el consentimiento de los menores transexuales para el inicio de operaciones y tratamientos de reasignación de sexo. Dado que algunos menores transexuales sienten esa pertenencia a un sexo diferente desde temprana edad y que mantienen ese sentimiento años después (el índice de permanencia), a día de hoy se aboga por la posibilidad de intervenciones previas a la mayoría de edad (Skougard, 2011: 1164). Este es un debate que excede nuestro análisis ${ }^{65}$. Nos puede ayudar el futuro pronunciamiento del TC sobre la cuestión de constitucionalidad que ha planteado el Tribunal Supremo (Auto 10-3-2016), en relación con el art. 1 de la Ley 3/2007 y la limitación de la legitimación para instar la rectificación de la mención registral de sexo a las personas mayores de edad. Ciertamente, al TC habrá de pronunciarse sobre una cuestión que afecta a los registros, y ahora estamos hablando de consentimiento en el ámbito de la salud, pero como el fundamento último es el derecho a esa identidad sexual, puede resultarnos útil. Dicho esto, el paralelismo con las pretensiones intersexuales solo puede ser parcial: aquella menor que nunca ha cuestionado el género asignado, cuyo cuadro cromosómico es acorde, pero cuyos órganos genitales tienen unas dimensiones excesivas para los estándares médicos, ha de poder instar la operación (e incluso solicitar el auxilio judicial si sus padres se

63 Véase Reynal Reillo (2017: 112-120), con un riguroso recorrido por la normativa autonómica.

64 Sobre la reforma, González Carrasco (2015: 1-5). Véase también Nevado (2017: 1544-1573), con una comparación del art. 9 antes y después de la reforma, y Sardinero García (2016: 207-221).

65 En Australia, el Re Alex, en el 2004, se centró en la petición de un transexual de 13 años que deseaba someterse a tratamiento hormonal. El tribunal estimó que no tenía la capacidad para consentir, ante la magnitud del tratamiento. Necesitaba el concurso de la intervención judicial. Véase Skougard (2011: 1164-1165) y Parlett y Weston-Scheuber (2004: 382 y ss.). En nuestro país, Vázquez-Pastor (2012: 715-728). 
opusieran) mucho más tempranamente. De pronto, edades como los 5 años de las sentencias del TC colombiano, que inicialmente desazonan, pueden entenderse mejor.

La FRA subrayó en el 2015 que los Estados europeos habían elaborado protocolos que abordaban la edad mínima recomendable para las operaciones (con el correlativo consentimiento de los menores): Suecia y Holanda, a partir de los 12 años. La FRA se hacía también eco de las dificultades de la pubertad para preguntarse si fijar en 15 años la edad adecuada (mencionaba Irlanda, Lituania, Polonia, Eslovenia y — ¿? - España), podía resultar excesivo (FRA, 2015: 7). Las edades son límites pretendidamente protectores, en un sentido u otro, pero el eje ha de ser la capacidad de cada menor, más cuando en el caso de los menores intersexuales el bagaje de su propia experiencia personal modelará necesariamente su decisión.

\section{EL ASESORAMIENTO CONTINUADO DE UN EQUIPO PLURIDISCIPLINAR}

Los análisis coinciden en aconsejar el counselling de un equipo inter-pluridisciplinar. Lo hemos visto en relación con el consentimiento, pero su función se proyecta más allá de la estricta formación de la voluntad. Vale la pena destacar el informe suizo, que aboga como medida prioritaria por un asesoramiento «expert, sensitive and individually tailored ${ }^{66}$, presente desde el diagnóstico hasta la madurez; también la Resolución 2191/2017 ${ }^{67}$.

La tarea de ese equipo no se centra en la operación, que puede ser un punto intermedio del trabajo conjunto, o incluso no tener lugar. Su primer cometido es crear un vínculo emocional normal entre los padres y el recién nacido ${ }^{68}$ y mentalizarlos de que esa situación no es una tragedia a superar. $\mathrm{Su}$ trabajo conjunto va a permitir que los progenitores encaren adecuadamente ese período de espera, en que se conforma la identidad sexual del menor, y les facilita calibrar las mejores opciones. Llama la atención cómo los análisis,

66 Swiss National Advisory Commission on Biomedical Ethics (2012: 18).

67 «7.1.3. provide all intersex people with health care offered by a specialized, multidisciplinary team taking a holistic and patient-centred approach and comprising not only medical professionals but also other relevant professionals such as psychologists, social workers and ethicists, and based on guidelines developed together by intersex organisations and the professionals concerned».

68 Recuerda la FRA: «The initial aim of Counselling and support is therefore to create a protected space for parents and the newborn, so as to facilitate a close bond. In addition, the parents need to be enabled to take the necessary decisions on the child's behalf calmly and after due reflection. In this process, they should not be subjected to time or social pressures» (FRA, 2015: 10). 
pese a constatar la utilidad de los equipos en relación con el bienestar de los menores intersexuales, insisten en el acompañamiento a las familias como tales.

\section{LA REGULACIÓN DE MALTA}

Malta se ha convertido en el primer país del mundo que aprueba una legislación integral prohibiendo las cirugías genitales, médicamente innecesarias y sin consentimiento, en menores de edad intersexuales $(2015)^{69}$. La Gender Identity, Gender Expression and Sex Characteristics Act adopta la Resolución 1952/2013 y menciona en su EM los informes de la ONU y de las instituciones europeas.

La ley reconoce a toda persona residente en Malta el derecho a la identidad de género y a su libre desarrollo sin necesidad de tratamientos quirúrgicos, hormonales o psiquiátricos (art. 3). Es un procedimiento simplificado de reconocimiento del género ante notario (o judicial en caso de los menores) dirigido a los transexuales; supone una simple declaración de autodeterminación, prohibiéndose cualquier solicitud de información médica. Además, se permite posponer la inclusión del sexo en el certificado de nacimiento hasta los 14 años, para posibilitar que sea el menor quien decida su identidad: hombre, mujer o neutro (arts. 8, 10 y 21).

69 Art. 14 (1): «It shall be unlawful for medical practitioners or other professionals to conduct any sex assignment treatment and/or surgical intervention on the sex characteristics of a minor which treatment and/or intervention can be deferred until the person to be treated can provide informed consent: Provided that such sex assignment treatment and/or surgical intervention on the sex characteristics of the minor shall be conducted if the minor gives informed consent through the person exercising parental authority or the tutor of the minor. // (2) In exceptional circumstances treatment may be effected once agreement is reached between the interdisciplinary team and the persons exercising parental authority or tutor of the minor who is still unable to provide consent: Provided that medical intervention which is driven by social factors without the consent of the minor, will be in violation of this Act. // (3) The interdisciplinary team shall be appointed by the Minister for a period of three years which period may be renewed for another period of three years. (4) The interdisciplinary team shall be composed of those professionals whom the Minister considers as appropriate. (5) When the decision for treatment is being expressed by a minor with the consent of the persons exercising parental authority or the tutor of the minor, the medical professionals shall: (a) ensure that the best interests of the child as expressed in the Convention on the Rights of the Child be the paramount consideration; and (b) give weight to the views of the minor having regard to the minor's age and maturity». 


\section{LA INSCRIPCION EN LOS REGISTROS ¿̇ES FACTIBLE (Y BENÉFICO) SUPERAR LA CLASIFICACIÓN BINARIA EN EL ÁMBITO ADMINISTRATIVO?}

\section{EL REFRENDO REGISTRAL URGENTE}

La necesidad de inscribir al recién nacido en un registro en un plazo relativamente breve, que imponen la práctica totalidad de los ordenamientos jurídicos, es un factor de presión para la realización de las operaciones correctoras. En buena medida, ha sido la conjunción de la necesidad de inscribir y la existencia de los avances médicos, con la posibilidad quirúrgica de borrar la ambigüedad, los que han transformado el problema. Históricamente, el derecho sí era consciente de los intersexuales, quienes durante el Renacimiento y la Edad Moderna pudieron escoger su sexo, aunque su declaración no era reversible $^{70}$. Baste recordar los Codex Maximilianeus Bavaricus Civilis y Allgemeine Landrecht prusiano ${ }^{71}$, que les permitían decidir por sí mismos ${ }^{72}$. Y tenemos ejemplos recientes: en 1970, el art. 1:17(2) CC holandés estableció que en caso de sexo incierto se podía poner en el certificado de nacimiento "sexo desconocido", una entrada modificable con posterioridad a mujer/ hombre (aunque el cambio permanecía visible en dicho certificado). En la práctica, padres y funcionarios - inquietos - pospusieron el registro, y ante la indeseada situación se reformó en 1995, diferenciando entre un certificado preliminar (con la incertidumbre) y uno definitivo, tres meses después, en que se fijaba el sexo o — todavía - se mantenía la indefinición. Para el legislador holandés, tres meses bastaban para fijar el sexo (Brink et al., 2015:285-286): una prueba más de los esfuerzos por cohonestar plasmación administrativa y salvaguarda de excepcionalidades; como veremos más adelante, la administración francesa imagina un plazo máximo de uno o dos años.

70 Chau y Heering (2002: 334); Greenberg (1999: 277-278).

71 El ALR [Der Zwitterparagraph] establece: «\$19. Cuando nace un hermafrodita son los padres los que determinan el sexo sobre la base del que recibirá su educación. // $\$ 20$. No obstante, una vez alcanzados los 18 años, le corresponde a la persona de aquellas características la libre elección de determinar a qué sexo pertenece. // $\$ 21$. Conforme a esta elección quedarán perfilados sus derechos en el futuro. $/ / \$ 22$. Si los derechos de un tercero dependen de un presunto hermafrodita, el primero podrá solicitar una investigación por un perito.// $\$ 23$. El dictamen del perito es el determinante, incluso contra la elección del hermafrodita y la de sus padres» (Wacke: 1990: 697). Los 18 años no se correspondían con la mayoría de edad prusiana (24 años, ALR I\$ 26), sino con la madurez matrimonial del hombre (II $1 \$ 37$ ).

72 El BGB no lo contempla, pero sí los comentarios que lo acompañan (Rolker, 2015). 
En este escenario, parecen aconsejables procedimientos de modificación registral especialmente simplificados en relación con los intersexuales: constituirían una correlación con esa asignación provisional de un sexo que se propone como alternativa a las operaciones «urgentes». También cabría fijar períodos de espera en relación con la identificación sexual, posibilitando que el propio afectado decida (Scherpe, 2011-2012: 112), aunque entonces, ¿no sería mejor imponerlos incluso en casos no ambiguos, más cuando en algunos supuestos la intersexualidad se manifiesta después? Pero su concreción reglamentaria es más compleja: si durante un tiempo la adscripción a un sexo queda en stand by, ¿cómo identificarlo en el ínterin? En la inscripción del nacimiento, todavía una casilla avala la distinción binaria hombre/mujer. ¿Cabe añadir una tercera casilla? Esa inclusión entronca con la reivindicación de un «tercer sexo" que defiende algún colectivo intersexual - y colectivos trans - ${ }^{73}$ y que objetan otros, que temen que puede incentivar las operaciones. ¿Un tercer sexo legal, como revolución copernicana?

\section{LA REGULACIÓN ESPAÑOLA}

En España, la inscripción de nacimiento — que debe realizarse en el plazo de ocho días — «hace fe [...] el sexo [...] del inscrito» (art. 41 LRC). En relación con los intersexuales, el art. 93.2 LRC establece que cabe rectificar la inscripción mediante expediente gubernativo — sin, por tanto, recurrir a los tribunalesen caso de «indicación equivocada del sexo cuando igualmente no haya duda sobre la identidad del nacido por las demás circunstancias» (en caso, por tanto, de inscripción no coincidente con el sexo asignado después). Este supuesto aparece desde $1957^{74}$ y se completó con «la mención registral relativa al sexo de las personas en los casos de disforia de género» (los transexuales), con la reforma de la Ley 3/2007, de 15 de marzo. Por tanto, los estados intersexuales son considerados, en relación con el sistema de acceso al Registro Civil, como errores originarios, rectificables mediante expediente gubernativo ${ }^{75}$. Esta puntualización es útil para deslindar transexuales e intersexuales: si los primeros pretenden cambiar su sexo inscrito, los segundos pretenden corregirlo ${ }^{76}$. Es una posible

73 Ben-Asher (2006: 69-72). Critica a la ISNA, por no cuestionar la dualidad.

74 La RDGRN 17.3.1982 aclaró que el art 93.2 LRC no era aplicable al supuesto de cambio de sexo como consecuencia de una intervención quirúrgica practicada a un transexual, pues en ese caso no hay error en la consignación del sexo del nacido al extenderse la inscripción de nacimiento, sino un cambio ulterior (que requería, entonces, sentencia judicial) [Cfr. Luces Gil: 2002, 267].

75 Benavente Moreda (2013a: 26).

76 Brink et al. (2015: 285). 
lectura del art. $307 \mathrm{RRC}$ cuando alude a los casos de modificación y rectificación de sexo (antes, los arts. 21.2 a y $22.2^{\circ}$ mencionan únicamente la rectificación de sexo). Finalmente, el art. 313 RRC estipula que «en caso de duda sobre el sexo o edad del nacido, emitirá dictamen el médico del registro civil o su sustituto». En la LRC del 2011 que entrará en vigor en los próximos años, se estima hecho inscribible "el sexo y el cambio de sexo" (art. $4.4^{\circ}$, véanse también los arts. 44, 49) y se explicita que la rectificación de sexo (art. 83.1.c), que debe vincularse al art. 91 — pensado para transexuales—, es un dato de publicidad restringida. Los intersexuales, como tales, permanecen invisibles.

Ya hemos mencionado dos proposiciones de ley $(2017,2018)$ que persiguen una clara relajación del procedimiento de rectificación registral, basado en la voluntad del solicitante. La del 2017, del Grupo Parlamentario Socialista, afirma que debe reformarse la Ley 3/2007 para «el reconocimiento del sexo registral de las personas intersexuales» (EM, párr. 3 in fine) y añade (EM, párr. 10):

[...] se aprovecha esta reforma para reconocer expresamente como beneficiarios y beneficiarias de esta ley a las personas intersexuales a las que en el momento de la inscripción registral se les asignó un sexo no concordante con el propio sexo sentido. Es obvio que realizándose dicha inscripción registral en los días posteriores a su nacimiento, resulta imposible conocer en ese momento cuál va a ser el sexo sentido por la persona recién nacida.

En realidad, la proposición subsume a los intersexuales en las soluciones de la rectificación ágil, sin un trato específico. No propone posponer la inscripción, sino facilitar los cambios a posteriori. Ciertamente, un intersexual puede sentir un sexo diferente del inscrito, pero no como opción, sino como consecuencia de su crecimiento. En el sentir de las proposiciones, no parece necesario individualizarlo.

\section{ALGUNAS SOLUCIONES DE DERECHO COMPARADO}

Frente a la tría de la condición hombre/mujer, la opción de la tercera categoría o casilla en la documentación administrativa y en los registros va paulatinamente consolidándose a nivel internacional. Es el criterio de la India, ${ }^{77}$

\footnotetext{
Pero el legislador hindú piensa en realidad en la comunidad de los hijras, que se autoconsideran de género femenino: «So that they no longer have to break their gender performance and dress as men to match their sex identifier on their passports when travelling through airports» (Hupf, 2015: 93).
} 
Nepal, ${ }^{78}$ Sudáfrica, Malasia, Nueva Zelanda ${ }^{79}$ o Australia —aquí a partir de la resolución de su Tribunal Supremo del 2 de abril de 2014 en el asunto NSW Register of Births, Deaths and Marriages $v$. Norrie, que admitió por primera vez en su historia mencionar en el registro civil el "sexo no específico»" ${ }^{80}$. Como último ejemplo, la Gender Recognition Act de California de 2017 —en vigor desde el 1 de septiembre de 2018 - introduce, pensando en los intersexuales, el tipo "no binario" ${ }^{81}$, como opción elegible (por ejemplo en los carnets de conducir). En nuestro país, la proposición de ley del 2018, cuando propone fijar un "sexo no binario», va en esa línea.

\section{UNA EFÍMERA ATENCIÓN A UN TERCER SEXO EN FRANCIA: LA SENTENCIA DEL TRIBUNAL DE TOURS DE 20 DE AGOSTO DEL 2015}

En Francia, el art. 58 Code fija la necesidad de mencionar si se está ante un hombre o una mujer, lo que no obsta para que ya tempranamente se discutiera: a finales del siglo XIX. Lacassagne —uno de los fundadores de la medicina legal- proponía reformar el precepto "pour imposer un examen médical à la puberté qui statuera le sexe et l'inscription comme homme, femme ou neutre sur les registres de l'état civil» (Lacassagne, 1887: 91). Ciertamente, Francia había apostado firmemente por la división binaria. Baste situar la Instruction générale relative à l'état civil, de 1999, aunque la firmeza hubo de modularse respecto de los transexuales, a partir de las condenas del TEDH.

En la lógica de asignar un sexo legal a las personas intersexuales, el art. 55 de la Circular del 28 de octubre de 2011 «relative aux règles particulières à divers actes de l'état civil relatifs à la naissance et à la filiation» ${ }^{82}$ establece:

78 Bochenek y Knight (2012: 11-41).

79 En abril del 2007 existían, de acuerdo con la información del Departamento del Interior, unos cuatrocientos pasaportes con X. Una pormenorizada información en FRA (2015: 4-5).

80 Bennett (2014: 847-873). Moron-Puech la critica porque: «Cette troisième catégorie apparaît donc subsidiaire par rapport deux autres [...] le pouvoir des médecins et le regard pathologique porté sur les personnes entreprenant une procédure de conformation sexuée n'est nullement remis en question, puisque l'accès au sexe "non spécifique" nécessite deux certificats médicaux» (Moron-Puech, 2014: 4).

81 «Sect. 2. (C) An option of a nonbinary gender designation on state-issued identification documents would allow intersex people, like transgender and nonbinary people, to be able to use state-issued identification documents that accurately recognize their gender identification as female, male, or nonbinary».

82 Accesible en: http://circulaires.legifrance.gouv.fr/pdf/2011/11/cir_34124.pdf. 
Lorsque le sexe d'un nouveau-né est incertain, il convient d'éviter de porter l'indication "de sexe indéterminé » dans son acte de naissance. Il y a lieu de conseiller aux parents de se renseigner auprès de leur médecin pour savoir quel est le sexe qui apparaît le plus probable compte tenu, le cas échéant, des résultats prévisibles d'un traitement médical. Ce sexe sera indiqué dans l'acte, l'indication sera, le cas échéant, rectifiée judiciairement par la suite en cas d'erreur.

Si, dans certains cas exceptionnels, le médecin estime ne pouvoir immédiatement donner aucune indication sur le sexe probable d'un nouveau-né, mais si ce sexe peut être déterminé définitivement, dans un délai d'un ou deux ans, à la suite de traitements appropriés, il pourrait être admis, avec l'accord du procureur de la République, quaucune mention sur le sexe de l'enfant ne soit initialement inscrite dans l'acte de naissance. Dans une telle hypothèse, il convient de prendre toutes mesures utiles pour que, par la suite, l'acte de naissance puisse être effectivement complété par décision judiciaire.

Pese a lo apuntado, la sentencia del Tribunal de Grande Instance de Tours del 20 agosto de 2015 admitió por primera vez la posibilidad de inscribir un sexo «neutro» (con el consiguiente revuelo en la prensa generalista y en los debates académicos). El caso era el siguiente: Jean-Pierre fue considerado de sexo masculino al nacer aunque su sexo no podía definirse de manera inequívoca. Cumplidos los sesenta, casado y con un hijo adoptado, Jean-Pierre afirmó que no se sentía capaz de definirse sexualmente y que debía constar su «identidad intersexual». Declaró en el juicio que su ambigüedad sexual lo había desestabilizado gravemente a lo largo de su existencia: a los 22 años los médicos habían reevaluado su asignación sexual y hasta los 35 su apariencia había sido «más bien femenina»; entonces, para prevenir la osteoporosis, había seguido un tratamiento hormonal que le había procurado una artificiosa apariencia masculina. Pretendía que constase en el Registro Civil su sexo neutro, dado que los formularios de algunos hospitales admitían una tercera opción. Para el TGI: «le sexe qui [lui] a été assigné à sa naissance apparaît comme une pure fiction (...) imposée durant toute son existence [...] il ne s'agit aucunement de reconnaître l'existence d'un quelconque "troisième sexe" mais de prendre acte de l'impossibilité de rattacher l'intéressé à tel ou tel sexe».

El 21 de marzo de 2016, el Tribunal de Apelación de Orleans lo contesta: "Admettre la requête de Monsieur X reviendrait à reconnaître, sous couvert d'une simple rectification d'état civil, l'existence d'une autre catégorie sexuelle». La Sentencia de la Cour de Cassation del 4 de mayo de 2017 lo ratifica e incorpora una evaluación de la proporcionalidad de la medida:

[...] attendu que la loi française ne permet pas de faire figurer, dans les actes de l'état civil, l'indication d'un sexe autre que masculin ou féminin; 
Et attendu que, si l'identité sexuelle relève de la sphère protégée par l'article 8 de la Convention de sauvegarde des droits de l'homme et des libertés fondamentales, la dualité des énonciations relatives au sexe dans les actes de l'état civil poursuit un but légitime en ce qu'elle est nécessaire à l'organisation sociale et juridique, dont elle constitue un élément fondateur; que la reconnaissance par le juge d'un "sexe neutre" aurait des répercussions profondes sur les règles du droit français construites à partir de la binarité des sexes et impliquerait de nombreuses modifications législatives de coordination;

Que la cour d'appel, qui a constaté que D... avait, aux yeux des tiers, l'apparence et le comportement social d'une personne de sexe masculin, conformément à l'indication portée dans son acte de naissance, a pu en déduire, sans être tenue de le suivre dans le détail de son argumentation, que l'atteinte au droit au respect de sa vie privée n’était pas disproportionnée au regard du but légitime poursuivi.

El interés del caso radica en la inversión de la secuencia habitual: no estamos ante un recién nacido al que no se sepa qué sexo asignar, sino ante alguien que siempre había vivido de acuerdo con la división binaria y que solicitó al Registro Civil poner sexo neutro ${ }^{83}$. Finalmente, la controversia ha abierto un debate en Francia sobre la oportunidad de un tercer sexo legal, con posiciones encontradas.

\section{LA SENTENCIA DEL TC ALEMÁN (BVG) DE 8 DE NOVIEMBRE DE 2017: RECONOCER UNA TERCERA OPCIÓN}

La sentencia del BVG del 8 de noviembre de $2017^{84}$ nos atrevemos a pronosticar que determinará el debate. La ley de estado civil [Personenstandsgesetz] alemana, tras la reforma del 2013, había fijado — lo hemos visto- la posibilidad de no rellenar la casilla hombre/mujer. Ahora bien, para algunos intersexuales adultos era una medida insuficiente, porque querían ser reconocidos como tales y es esta la pretensión de la recurrente ante el TC alemán. Será atendida. El BVG afirma que la imposibilidad de tener una entrada positiva, diferente de los géneros femenino y masculino, esto es, la fijación por exclusión ajena a la división binaria del sexo del solicitante sin poder explicitar la condición "diversa», contesta el libre desarrollo de la personalidad del art. 2(1) GG, en conjunción con el art. 1(1) y el art. 3. Dado que el libre desarrollo protege la identidad de género de hombres y mujeres, ha de proteger también la de

83 Binet (2016, 50); Ancel (2016: 278).

84 Disponible, en inglés, en: www.bundesverfassungsgericht.de. 
quienes no se identifican con uno de ellos ${ }^{85}$. Correlativamente, el BVG insta al Gobierno a reformar la ley antes del 31 de diciembre 2018. Este cambio abre, creemos, la puerta a futuras reformas legislativas en ese sentido.

\section{INTERSEXUALES Y LEGISLACIÓN ANTIDISCRIMINATORIA}

\section{LA APUESTA DE LA UNIÓN EUROPEA}

La UE proscribe la discriminación en el art. 21.1 de la Carta de Derechos Fundamentales «por razón de sexo, [...] características genéticas, [...] nacimiento, discapacidad, edad u orientación sexual». Desde la voluntad de erradicar la discriminación entre hombres y mujeres, y contra transexuales, aprueba la Recomendación CM/Rec (2010) del Comité de Ministros a los Estados miembros sobre las medidas para combatir la discriminación por motivos de orientación sexual o identidad de género; las resoluciones del Parlamento Europeo de 8 de febrero de 1994, de 18 de enero de 2006 y de 24 de mayo de 2012, relativas a la igualdad de derechos de lesbianas y gais y a la lucha contra la discriminación y la homofobia; la resolución del Parlamento Europeo, de 4 de febrero de 2014, sobre la hoja de ruta de la UE contra la homofobia y la discriminación por motivos de orientación sexual e identidad de género o la Directiva 2000/78/CE, del Consejo, relativa al establecimiento de un marco general para la igualdad de trato en el empleo y la ocupación. Curiosamente, el Parlamento Europeo, en la Resolución de 15 de septiembre de 2016, sobre la aplicación de la Directiva 2000/78/CE del Consejo, menciona a los intersexuales - que no aparecían en la directiva- bajo el bloque (genérico e inexacto técnicamente) de «Orientación sexual» y: «68. [...] pide a la Comisión Europea y a los Estados miembros que tengan en

85 «39. [...] Under the given circumstances, the assignment of gender is of paramount importance for individual identity; it typically occupies a key position both in a person's self-image and in the way this person is perceived by others. Gender identity plays an important role in everyday life: In part, gender determines entitlements and obligations provided for by law; furthermore, it often forms the basis for the identification of a person, and gender identity is also significant in everyday life irrespective of legal provisions. To a large extent it determines, for instance, how persons are addressed or what is expected of a person in terms of their appearance, upbringing or behaviour. // 40. The gender identity of persons who can be assigned neither the male nor the female gender is protected as well. These persons might be able to develop their personality more freely if less significance was attributed to gender assignment in general». 
cuenta la discriminación específica que sufren las personas intersexuales en cuanto al empleo y que revisen sus leyes y prácticas con vistas a impedir la discriminación de las personas intersexuales».

Aquí nos interesa específicamente el estudio que encargó la DG de Justicia «Trans and intersex people» (2011), que hemos mencionado recurrentemente (Agius y Tobler, 2011). Aunque se centra en los transexuales (algo inevitable), dedica un último apartado a los intersexuales y reconoce: «It remains unclear whether intersex people are implicitly covered by the existing EU anti-discrimination legal framework. This is mainly because EU gender equality legislation is silent on the issue and the definition of the ground of sex is still based on the male/female binary sex model» (Agius y Tobler, 2011: 82).

Examina su correlación con los transexuales y recuerda que el índice para fijar las acciones antidiscriminatorias es la identidad de género, ajeno al colectivo intersexual (que no es una subcategoría del «trans umbrella term»). En definitiva: «Discrimination against intersex people is a particularly complex form of sex discrimination» (Agius y Tobler, 2011: 7) ${ }^{86}$.

\section{AUSTRALIA COMO AVANZADILLA: ¿̇UNA OPCIÓN A EMULAR?}

En Australia, la Sex Discrimination Amendment (Sexual Orientation, Gender Identity and Intersex Status) Act (2013) es la primera ley que los individualiza. Se apoya en la Sex Discrimination Act de 1984, que ya abordaba la discriminación de las personas intersexuales (sec. 5 C) ${ }^{87}$. Por tanto, la condición intersexual deviene una causa en sí misma, lo que justifica un trato independiente respecto de otros colectivos. Como se apunta en la memoria de la

86 Es el índice que defienden mayoritariamente las asociaciones intersexuales como óptimo para el ejercicio de acciones antidiscriminación.

87 «Discrimination on the ground of intersex status: (1) For the purposes of this Act, a person (the discriminator) discriminates against another person (the aggrieved person) on the ground of the aggrieved person's intersex status if, by reason of: (a) the aggrieved person's intersex status; or (b) a characteristic that appertains generally to persons of intersex status; or (c) a characteristic that is generally imputed to persons of intersex status; the discriminator treats the aggrieved person less favorably than, in circumstances that are the same or are not materially different, the discriminator treats or would treat a person who is not of intersex status. // (2) For the purposes of this Act, a person (the discriminator) discriminates against another person (the aggrieved person) on the ground of the aggrieved person's intersex status if the discriminator imposes, or proposes to impose, a condition, requirement or practice that has, or is likely to have, the effect of disadvantaging persons of intersex status». 
ley: "While there may be some overlap between the grounds of "sex", "gender identity" and "intersex status", it is important that intersex status is protected as a separate ground because people who are intersex are also vulnerable to discrimination. It also recognises that discrimination on this ground manifests differently to discrimination on the grounds of sex and gender identity».

Este parece el último eslabón de protección de la discriminación por razón de sexo o género ${ }^{88}$. Todavía es singular, pero procura una pista a seguir.

\section{INTERSEXUALES Y DISCAPACITADOS}

En EE. UU. se ha debatido si las personas intersexuales podrían constituir una categoría de discapacitados ${ }^{89}$, sujetos a la Americans with Disabilities Act (1990), a los efectos de su legitimación para actuar contra la discriminación. Desde nuestras coordenadas no parece una opción adecuada, pero identifica un interesante debate: para los movimientos en defensa de los derechos de los discapacitados, estos son capaces de una participación plena en sociedad, solo que no de manera equiparable a otros colectivos, al existir condiciones normativas que privilegian determinados cuerpos. El emparejamiento de intersexuales y discapacitados pretende desmitificar, como explicitan sus valedores, la idea del cuerpo de referencia único (Schmidt, 2013: 183).

\section{LAS LEYES AUTONÓMICAS DE TUTELA DE LOS LGTBI Y CONTRA LA DISCRIMINACIÓN}

En los últimos años se han multiplicado las leyes autonómicas que atienden al colectivo LGTB. La mayoría se hacen eco de los intersexuales (ergo LGTBI). A día de hoy contamos con doce leyes: Navarra, País Vasco, Galicia, Cataluña, Andalucía, Canarias, Extremadura, Madrid — que los imbrica en dos leyes-, Murcia, Islas Baleares y Valencia ${ }^{90}$. Comparten núcleo regulador

88 Gelfman hace la siguiente clasificación: «A. The First Generation: The "Plain Meaning” of Sex; B. The Second Generation: Sex Stereotyping; C. The Third Generation: Discrimination Against Transgender Individuals; D. Moving Forward: Implications for Intersex Individuals» (Gelfman, 2010: 73-84). Véase también Ezie (2011: 141200).

89 Menon (2011: 1221-1251); Das (2014: 21-34).

90 Ley foral 12/2009, de 19 de noviembre, de no discriminación por motivos de identidad de género y de reconocimiento de los derechos de las personas transexuales (BOE, núm. 307, 22-12-2009) — los menciona-; Ley del País Vasco 14/2012, de 28 de junio, de no discriminación por motivos de identidad de género y de reconocimiento 
y recurrentes alusiones a los Principios de Yogyakarta. Su pretensión última es garantizar, en el marco de las competencias autonómicas, los derechos de los LGTB[I] —de modo prioritario de los transexuales — en la sociedad, individualizando ámbitos de tuición como la educación, la salud, los medios de comunicación, el trabajo o el deporte. También persiguen luchar contra la discriminación, como denota, por ejemplo, la «Cláusula general antidiscriminatoria» del art. 5 de la Ley 12/2014 de Cataluña. En una progresiva depuración técnica, las leyes más recientes incorporan conceptos como discriminación directa, indirecta, múltiple, por asociación, por error, etc. ${ }^{91}$, así como el principio de inversión de la carga de la prueba en las acciones antidiscriminación.

de los derechos de las personas transexuales (BOE, núm. 172, de 19-7-2012); Ley gallega 2/2014, de 14 de abril, por la igualdad de trato y la no discriminación de lesbianas, gays, transexuales, bisexuales e intersexuales (BOE, num. 127, de 26-5-2014); Ley $2 / 2014$, de 8 de julio, integral para la no discriminación por motivos de identidad de género y reconocimiento de los derechos de las personas transexuales de Andalucía (BOE, núm. 183, de 9-8-2014) —no los contempla—; Ley 11/2014, de 10 de octubre, catalana, para garantizar los derechos de lesbianas, gays, bisexuales, transgéneros e intersexuales y para erradicar la homofobia, la bifobia y la transfobia (BOE, núm. 281, de 20-11-2014); Ley canaria 8/2014, de 28 de octubre, de no discriminación por motivos de identidad de género y de reconocimiento de los derechos de las personas transexuales (BOE, núm. 108, de 6-5-2015) —no los contempla—; Ley 12/2015, de 8 de abril, de igualdad social de lesbianas, gais, bisexuales, transexuales, transgénero e intersexuales y de políticas públicas contra la discriminación por orientación sexual e identidad de género en la Comunidad Autónoma de Extremadura (BOE, núm. 108, de 6-5-2015); Ley 2/2016, de 29 de marzo, de Identidad y Expresión de Género e Igualdad Social y no Discriminación de la Comunidad de Madrid (BOE, núm. 169, de 14-7-2016). Debe completarse con la Ley 3/2016, de 22 de julio, de protección integral contra la LGTBifobia y la Discriminación por Razón de Orientación e Identidad Sexual en la Comunidad de Madrid (BOCM, núm. 190, de 10-8-2016); Ley 8/2016, de 27 de mayo, de igualdad social de lesbianas, gais, bisexuales, transexuales, transgéneros e intersexuales, y de políticas públicas contra la discriminación por orientación sexual e identidad de género de la Comunidad autónoma de la región de Murcia (BOE, núm. 153, de 25-6-2016); Ley 8/2016, de 30 de mayo, de la Comunidad Autónoma de les Illes Balears, para garantizar los derechos de lesbianas, gays, trans, bisexuales e intersexuales y para erradicar la LGTBI fobia (BOE, núm. 157, de 30-6-2016) y Ley 8/2017, de 7 de abril, integral del reconocimiento del derecho a la identidad y a la expresión de género en la Comunitat Valenciana (BOE, núm. 112, de 11-5-2017) —tampoco los menciona-.

91 Así, el art. 4 de la Ley 8/2016 de Murcia, el art. 4 de la Ley12/2015 de Extremadura o el art. 3 de la Ley 3/2016 de Madrid. 
La atención a los intersexuales se advierte tangencial, aunque al cotejar las sucesivas leyes se constata un interés in crescendo. Intuimos una secuencia cronológica: si una primera ley los menciona de refilón, la siguiente se adhiere sin una reflexión excesiva, la que aparece meses después ya los hace suyos sin interrogantes y la más próxima en el tiempo — con un legislador más informado- incorpora una atención menos programática. Así, la ley navarra de 2009, una ley pionera, solo los nombra en la EM (párr. 6) para clarificar qué entiende por transexuales; luego la ley vasca de 2012 los integra en la regulación de los transexuales con una mención equívoca: parece considerarlos una subcategoría de aquellos ${ }^{92}$; después la ley gallega de 2014 les otorga una atención sesgada: en ocasiones aparecen en la relación LGTB; en otras, sencillamente se ignoran ${ }^{93}$.

La ley catalana de 2014 da un paso más, pues les dedica un título específico (el tercero: «Transidentidad e intersexualidad»), pese a que se incorporaron en la tramitación parlamentaria ${ }^{94}$. Se asocian con las personas transgénero. Vale la pena reproducir algunos apartados:

Artículo 23. Personas transgénero y personas intersexuales.

1. En el ámbito de las administraciones públicas de Cataluña, especialmente en el ámbito educativo y universitario, deben establecerse por reglamento las condiciones para que las personas transgénero y las personas intersexuales sean tratadas y nombradas de acuerdo con el nombre del género con el que se identifican, aunque sean menores de edad.

$[\ldots]$

3. Debe garantizarse, en cualquier caso, el derecho a consulta y a información específica para personas transgénero y para personas intersexuales en ámbitos como el acceso al mercado de trabajo, los tratamientos hormonales y las intervenciones quirúrgicas o la salud sexual y reproductiva.

92 Así, el art. 6.4 (Servicios de asesoramiento y apoyo a las personas transexuales, sus familiares y personas allegadas) concluye: «Los servicios referidos en el presente artículo atenderán también de forma específica a las personas intersexuales». Y punto.

93 Baste como ejemplo el art. 19.1: «La consejería competente en materia de sanidad garantizará la información sobre homosexualidad, bisexualidad y transexualidad que recoge la OMS a todas las personas que trabajan en el ámbito de la salud, tanto en el público como en el privado o concertado, de modo que se garantice que los profesionales del ámbito sanitario conozcan dicha información».

94 Lauroba (2015: 179-198); González Beilfuss (2015: 195-221). 
4. Las personas transgénero y las personas intersexuales deben poder acogerse a lo establecido por la presente ley sin necesidad de un diagnóstico de disforia de género ni tratamiento médico.

Es una regulación que, de nuevo, deriva hacia la declaración de intenciones: así el párr. 1, que remite a un reglamento ulterior como coartada para las inconcreciones. Pero la eliminación del diagnóstico de disforia de género conlleva la anhelada "despatologización». Más allá de discrepar de la correlación entre transgéneros e intersexuales, importa señalar, tout court, el art. 17 (Salud) como deber de las administraciones catalanas: «Velar porque la política sanitaria sea respetuosa hacia las personas LGBTI y no trate directa o indirectamente la condición de estas personas, especialmente transgéneros e intersexuales, como una patología».

Las leyes extremeña (2015), madrileñas -2 leyes, que en cierta medida se solapan- (2016), murciana (2016) y balear (2016) les prestan un mayor protagonismo. Hemos visto en el apdo. I de este artículo la definición de intersexual de la Ley 2/2016 de Madrid y vale la pena recuperar la «declaración de principios» de su EM — que en parecidos términos aparece en otras EM—:

La protección de las personas intersexuales exige el reconocimiento de la diversidad de los cuerpos humanos y la erradicación del prejuicio según el cual existe un único patrón normativo de corrección corporal, que lleva a que menores intersexuales sean operados en su infancia para asimilarlos al patrón normativo de hombre o mujer, sin saber cuál es la identidad de dicha persona y cometiendo con ello frecuentes errores que luego condicionan gravemente la vida de la persona intersexual. Sin conocimiento de la identidad de género sentida por la persona intersexual: hombre, mujer o simplemente intersexual, cualquier intervención quirúrgica que asimile al menor a una identidad puede ser una autentica castración traumática.

Se completa con la prohibición taxativa en el art. 4.3 de la Ley 2/2016 de «las cirugías genitales de las personas intersexuales que no obedezcan a la decisión de la propia persona afectada a la necesidad de asegurar una funcionalidad biológica por motivos de salud» y con la "Atención sanitaria a personas intersexuales» (art. 15.1) $)^{95}$.

95 «Atención sanitaria a personas intersexuales. 1. El sistema sanitario público de Madrid velará por la erradicación de las prácticas de modificación genital en bebes recién nacidos atendiendo únicamente a criterios quirúrgicos y en un momento en el que se desconoce cuál es la identidad real de la persona intersexual recién nacida. Todo ello con la salvedad de los criterios médicos basados en la protección de la salud de la persona recién nacida y con la autorización legal. // 2. Se procurará conservar las 
Como última manifestación, el título IV de la Ley balear 8/2016 contempla a las "Personas intersexuales» y permite intuir un conocimiento real de su problemática:

Artículo 23. Protocolo de atención integral a personas intersexuales.

1. Se establecerá un protocolo específico de actuación en materia de intersexualidad que incluirá la atención psicológica adecuada a la persona intersexual y a los padres/ madres o personas tutoras, y los tratamientos requeridos en atención al género con el cual se identifica.

Se evitará siempre que sea posible la intervención médica inmediata (quirúrgica u hormonal) del proceso de normalización sexual para ajustarse a las normas físicas del binarismo de género.

2. El sistema sanitario público de las Illes Balears velará por la erradicación de las prácticas de modificación genital en bebés recién nacidos atendiendo únicamente a criterios quirúrgicos y en un momento en el cual se desconoce cuál es la identidad real de la persona intersexual recién nacida. Todo ello con la excepción de los criterios médicos basados en la protección de la salud de la persona recién nacida.

3. Se procurará conservar las gónadas con el fin de preservar una futura aportación hormonal no inducida, incluyendo en los controles los marcadores tumorales.

4. No se realizarán pruebas de hormonación inducida con finalidades experimentales ni de otro tipo hasta que la propia persona o sus tutores legales así lo requieran en función de la identidad sexual con la cual se identifica.

5. Se formará al personal sanitario haciendo especial hincapié en la corrección de trato y la privacidad.

6. Se preservará la intimidad del paciente en su historia clínica de forma que no todo el personal sanitario pueda conocer su condición, excepto cuando sea estrictamente necesario.

gónadas con el fin de preservar un futuro aporte hormonal no inducido. Incluyendo en los controles los marcadores tumorales. // 3. No se realizarán pruebas de hormonación inducida con fines experimentales ni de otro tipo hasta que la propia persona o sus tutores legales, así lo requieran en función de la identidad sexual sentida». // 4. «Se limitarán las exploraciones genitales a lo estrictamente necesario y siempre por criterios de salud. // 5. Se formará al personal sanitario haciendo especial hincapié en la corrección de trato y la privacidad.// 6. Se preservará la intimidad del paciente en su historia clínica de manera que no todo el personal sanitario que acceda a ella pueda conocer su cambio de identidad de género, salvo cuando sea estrictamente necesario». 
Podemos lamentar que la intersexualidad, aunque progresivamente presente, es colateral: lo avala que se ignore la intersexofobia, pero aun así constituyen un esfuerzo normativo, conceptual y pedagógico útil para proteger al colectivo.

\section{ALGUNAS REFLEXIONES}

Querríamos pensar que, a estas alturas, hemos contribuido a situar las principales cuestiones jurídicas que afectan a las personas intersexuales ${ }^{96}$ ¿Qué resulta clave, a día de hoy? Sin lugar a dudas, la salvaguarda de sus derechos fundamentales. Dicho esto, se tiene la impresión de que, al descartarse la necesidad ("emergencia») de las operaciones tempranas, el problema jurídico no es complejo. En definitiva, se está produciendo un cambio de paradigma al pasar de concebir la operación como la opción que mejor protege el interés superior del menor a entender que la mejor manera de atender dicho interés es posponiéndola. Se debe optar por la moratoria, pero no como un principio indeclinable, sino como punto de partida que la singularidad de cada supuesto puede contestar, con la posibilidad de intervenciones durante la minoría de edad si el menor intersexual, con el conocimiento adecuado, así lo decide ${ }^{97}$.

La otra gran cuestión es la plasmación en los registros civiles. Podemos buscar fórmulas administrativas que atiendan a los intereses en juego: lapsos más largos para inscribir, mecanismos alternativos... Ante el dilema sobre la inclusión de otra casilla a efectos administrativos, uno se pregunta si no podríamos tout court eliminar las casillas. Como recuerda la Resolución 2191/2017, «[7.3.4]. Consider making the registration of sex on birth certificates and other identity documents optional for everyone». No parece descabellado priorizar otros mecanismos identificativos de las personas, léase el reconocimiento facial, el iris o las huellas digitales (Mcgrath, 2009: 370) ${ }^{98}$.

Las construcciones técnicas nos abocan a un debate de mayor enjundia sobre la admisión de un tercer sexo legal, con el plus de la sentencia del BVG,

\footnotetext{
96 Quedan materias que merecerían una contribución autónoma, como la problemática jurídica de los atletas, que mencionamos al inicio. Otras han sido superadas por la evolución legislativa de los Estados, como el ius connubii.

97 En cierta medida, avalan esta interpretación a posteriori recientes reclamaciones judiciales de personas intersexuales en Alemania (Christiane Völling) y EE. UU. (el caso Crawford). Véase White (2014: 805-807).

98 Véase también Reilly (2005: 318).
} 
que tendrá — creemos - un efecto reflejo en breve. Ahora bien, reconocer un tercer sexo no es necesariamente una panacea: podría constituir un nuevo elemento de discriminación ${ }^{99} \mathrm{y}$ acaso una división trimembre resulte también restrictiva ${ }^{100}$. Por ello, en ese abanico de realidades individuales, se ha propuesto una «escala de identidad sexual», donde los sujetos se ubicarían en los diferentes peldaños. Esta opción, se aduce, daría lugar a cambios legales de mayor envergadura que aceptar un tercer sexo jurídico (o non-binary gender, en expresión de la ley californiana o de la proposición de ley del 2018) puesto que, «en lugar de hombres y mujeres, la ley tendría que tratar a cada individuo como persona» (Chau y Herring, 2002: 357). Es una reflexión sugerente. Y que puede intuirse en informes recientes, como el de HRW-InterACT: «We are taught that sex is dimorphic: simply male or female. But sex, in reality, is a spectrum - with the majority of humans appearing to exist at one end or the other. In fact, as many as 1.7 percent of babies are different from what is typically called a boy or a girl» (HRW-InterACT, 2017: 5).

¿Espectro? ¿Escala? Dado que el siglo XXI se anuncia como un universo VUCA en que incluso las ideas jurídicas inmutables se resquebrajan como los espejos de la última escena de La dama de Shanghái, creemos estimulante apuntar esta idea, y continuar, después, el debate.

99 «Je me méfie d'une proposition comme celle allant dans la direction d'une troisième catégorie pour placer les phénomènes qui ne sont pas classés comme féminin-masculin selon les critères traditionnels. Je me méfie de cette proposition qui a été écartée par les juristes, pour plusieurs raisons. Il s'agit d'un côté d'une tentative de renfermer dans un ghetto ce type d'identité sexuelle; c'est en même temps une tentative pour pas reconnaître une réalité nouvelle, la façon nouvelle de considérer le problème de l'identité sexuelle qui nous amène à une reconsidération générale du problème, et c'est du point de vue théorique une attitude conservatrice, car il s'agit d une tentative de ne pas mettre en discussion la polarité traditionnelle et d'ajouter aux catégories déjà connues une troisième et nouvelle catégorie» (Rodotà, 1995: 19). Más reciente, Peron: «L'admission d'un troisième genre (genre neutre) ne serait-ce pas persister dans la classification des individus, et par suite, être porteur de discrimination? La solution la plus simple n'est-elle pas de nier toute distinction de genre, et d'éliminer le critère du sexe du droit?» (Peron, 2015: 4).

100 "Once one accepts the argument that the male-female dyad is a social construction, which unreasonably restricts people's sexual identity into one of two sexes, it becomes hard to deny that restricting people to three identities is open to identical objections» (Chau y Herring, 2002: 356). Como recordatorio — itan citado!- - los cinco sexos que enumera Fausto-Sterling (2000: 17-23). 


\section{Bibliografía}

Agius, S. (2015). Human Rights and intersex People. Council of Europe. Disponible en: https://rm.coe.int/16806da5d4.

- y Tobler, C. (2011). Trans and intersex people: Discrimination on the grounds of sex, gender identity and gender expression. Report by the European Network of Legal Experts in the non-discrimination field, Brussels.

Agón López. J. G. (2017). Consentimiento informado y responsabilidad médica. Madrid: La Ley-Wolters Kluwer.

Aliabadi, S. A. (2005). You Make Me Feel like a Natural Woman: Allowing Parents to Consent to Early Gender Assignment Surgeries for their Intersexed Infants. William \& Mary Journal of Women and the Law, 11, 427-459.

Ancel, B. (2016). Un OJNI (objet juridique non identifié) à l'état civil. Boîte de Pandore ou révolution juridique à l'échelle mondiale? Semaine juridique, 11, 278-284.

Asensio Sánchez, M. A. (2012). Patria potestad, minoría de edad y Derecho a la salud. Madrid: Pluconde-Dykinson.

Ben-Asher, N. (2006). The necessity of sex change: a struggle for intersex and transex liberties. Harvard Journal of Law\& Gender, 29, 69-72.

Benavente Moreda, P. (2013a). Orientación sexual e identidad de género y relaciones jurídico privadas. Revista General de Derecho Constitucional, 17, 1-75.

— (2013b). Identidad y contexto inmediato de la persona (identidad personal, el nombre de la persona, identidad sexual y su protección. Anuario de la Facultad de Derecho de la Universidad Autónoma de Madrid, 5-161.

Bennett, Th. (2014). "No man's land": non-binary sex identification in Australian Law and Policy. UNSW Law Journal, 37, 847-873.

Benson, S. R. (2005). Hacking the Gender Binary Myth: Recognizing Fundamental Rights for the Intersexed. Cardozo Journal Law and Gender, 12, 31-63.

Berghausen, M. (2011). Intersex Employment Discrimination: Title VII and Anatomical Sex Nonconformity. Northwestern University Law Review, 105, 1281-1315.

Binet, J. R. (2016). Sexe neutre: un utile rappel à la loi, Droit de la Famille, 5, 50-51.

Bird, J. (2005). Outside the Law: Intersex, Medicine and the Discourse of Rights. Cardozo Journal Law and Gender, 12, 65-80.

Bishop, E. A. (2007). A Child's Expertise: Establishing Statutory Protection for Intersexed Children who reject their Gender of Assignment. New York University Law Review, 82, 531-568.

Bochenek, M. y Knight, K. (2012). Establishing a Third Gender Category in Nepal: process and Prognosis. Emory Int'l L.Rev, 26, 11-41.

Brink, M., Reu $\beta$, P. y Tigchelaar, J. (2015). Out of the Box? Domestic and Private International Law Aspects of Gender Registration. A Comparative Analysis of Germany and the Netherlands. European Journal of Law Reform, 17, 282-293.

Chau, P. L. y Herring, J. (2002). Defining, Assigning and Designing Sex. International Journal of Law, Policy and the Family, 16, 327-367. 
Curtis, S. (2011). Reproductive Organs and Differences of Sex Development: The Constitutional Issues Created by the Surgical Treatment of Intersex Children. McGeorge Law Review, 42, 841-872.

Das, A. (2014). Paper Presented at the Conference A Bio-Political Perspective on Intersexuality and Disability In Discourses Of Law. Socio-Legal Review, 10, 21-34.

Davidian, A. (2011). Beyond the locker Room: Changing Narratives on early Surgery for Intersex Children. Wisconsin Journal of Law, Gender and Society, 26, 1-26.

Deutsche Ethikrat. (2013). Intersexuality. Opinion. Disponible en: https://bit.ly/2I8pINv. Eekeelar, J. (2006), Family Law and Personal Life. Oxford: Oxford University Press.

Ezie, C. (2011). Deconstructing the Body: Transgender and Intersex identities and Sex Discrimination. The Need of a Strict Scrutiny. Columbia Journal of Gender and Law, 20, 141-200.

Fausto-Sterling, A. (2000). The Five Sexes, revisited: The Varieties of Sex Will Test Medical Values and Social Norms. The Sciences, 40 (4), 17-24.

Ford, K. K. (2001). "First, Do No Harm". The Fiction of Legal Parental Consent to Genital-Normalizing Surgery on Intersexed Infants. Yale Law and Policy Review, 19, 469-488.

FRA (European Union Agency for Fundamental Rights). (2015). The fundamental rights situation of intersex people. Disponible en: https://bit.ly/1fDvPdG.

García López, D. (2015). La intersexualidad en el discurso médico-jurídico. Eunomía. Revista en Cultura de la Legalidad, 8, 54-70.

Gelfman, I. (2010). Because of Intersex: Intersexuality, Title VII, and the Reality of Discrimination "Because of... [Perceived] Sex". New York University Review Law and Social Change, 34, 55-122.

Ghattas, D. C. (2013). Human Rights between the Sexes. A preliminary Study on the life situations of inter* individuals. Heinrich Böll Stiftung. Disponible en: https://bit. ly/2I8qiLb.

Glazer, S. (2011). Sporting Chance: Litigating Sexism out of the Olympic Intersex policy. Journal Law and Policy, 20, 545-580.

González Beilfuss, M. (2015). El reconocimiento jurídico de la diversidad sexual en la España de las autonomías: la Ley 11/2014 del Parlamento de Cataluña. Istituzioni del Federalismo, 1, 195-221.

González Carrasco, M. C. (2015). La (adecuada) limitación de la autonomía sanitaria del menor introducida por la ley 26/2015, de 28 de julio, de modificación del sistema de protección a la infancia y la adolescencia. Disponible en: https://bit.ly/2I8qH0o.

González Sánchez, P., Velásquez Acevedo, C. y Duque Quintero, S. P. (2010). Problemática jurídica de los estados intersexuales. El caso colombiano. Iatreia, 23, 204-211.

Greenberg, J. A. (1999). Defining Male and Female: Intersexuality and the Collision between Law and Biology. Arizona Law Review, 41, 265-92.

(2002). What do Scalia and Thomas really think about Sex? Title VII and gender nonconformity Discrimination: Protection for Transsexuals, Intersexuals, Gays and Lesbians. Thomas Jefferson Law Review, 149-159.

- (2011). Health Care Issues Affecting People with an Intersex Condition or DSD: Sex or Disability Discrimination? Loyola of Los Angeles. Law Review, 45, 849-908. 
Hammarberg, T. (2010). Derechos humanos e identidad de género. Disponible en: https:// bit.ly/2wGGZfy.

Hermer, L. (2002). Paradigms revised: Intersex Children, Bioethics and Law. Annals of Health Law, 195-236.

- (2007). A moratorium on intersex surgeries? Law, Science, Identity, and bioethics at the crossroads. Cardozo Journal of Law and Gender, 13, 255-272.

Hester, J. D. (2004). Intersex(es) and informed consent: how physicians' Rhetoric constrains choice. Theoretical Medicine, 25, 21-49.

Holmes, M. (2005). Distracted Attentions: Intersexuality and Human Rights Protections. Cardozo Journal of Law and Gender, 12, 127-133.

Human Rights Watch-InterACT. (2017). I Want to Be Like Nature Made Me. Medically Unnecessary Surgeries on Intersex Children in the US. Disponible en: https://www. hrw.org/.

Hupf, R. (2015). Allyship to the Intersex Community on Cosmetic Non-Consensual Genital 'normalizing' Surgery. William and Mary Journal of Women and the Law, 22, 73-104.

Kismödi, E. y Shakespeare, T. (2014). Eliminating forced, coercive and otherwise involuntary sterilization An interagency statement. World Health Organization. Disponible en: https://bit.ly/2jNkf4t.

Köllen, T. (2016). Intersexuality and Trans-Identities within the Diversity Management Discourse. En T. Köllen (ed.). Sexual Orientation and Transgender Issues in Organisations-Global Perspectives on LGBT Workforce Diversity (pp. 1-20). Heidelberg, Cahm, New York: Springer.

Kraus, C. (2015). Classifying Intersex in DSM-5: Critical Reflections on Gender Dysphoria. Archives of sexual behavior, 44, 1147-1163.

Lacassagne, A. (1887). Les actes de l'état civil. Paris: A. Starck.

Lareau. A. C. (2003). Who decides? Genital normalizing surgery on intersexed infants. Georgetown Law Journal, 92, 129-151.

Lauroba, E. (2015). La Ley catalana 11/2014 para garantizar los derechos de los LGBTI y erradicar la homofobia, la bifobia y la transfobia: ¿una declaración de intenciones bienintencionada o una norma de efectos jurídicos reales? GenIus, 2015/2, 179198.

Lisdonk, J. V. (2014). Living with Intersex/DSD. An Exploratory Study of the Social Situation of Persons with intersex/DSD. The Hague: Netherlands Institut of Social Research.

Luces Gil, F. (2002). Derecho del Registro civil. Barcelona: Bosch.

Lloyd, E. (2005). From the Hospital to the Courtroom: A Statutory Proposal for Recognizing and Protecting the Legal Rights of Intersex Children. Cardozo Journal Law and Gender, 12, 156-195.

Mcgrath, J. (2009). Are you a Boy or a Girl? Show me your real ID. Nevada Law Journal, 9 (2), 368-406.

Menon, Y. (2011). The Intersex Community and the Americans with Disabilities Act. Connecticut Law Review, 43, 1221-1251. 
Miano, P. (2015). Intersex condition and the construction of gender identity. International Journal of Humanities and Cultural Studies, 2, 586-598.

Morland, I. (2014). Intersex. Transgender Studies Quarterly,1 (1-2), 111-115.

Moron-Puech, B. (2014). Création d'un sexe non spécifique par la Haute Cour d'Australie. Mention du sexe à l'état civil (Australie). La Revue des droits de l'homme, 1-6.

Muckle, C. (2006). Giving a Voice to Intersex Individuals through Hospital Ethics Committees. Wisconsin Law Review, 2006/3, 987-1023.

Nevado, V. (2017). El interés superior del menor maduro en situación de grave riesgo: entre la autonomía del paciente y el derecho a la vida. Anuario de Derecho Civil, 70 (4), 1544-1573.

Newcombe, P. (2017). Blurred Lines-Intersexuality and the Law: An Annotated Bibliography. Law Library Journal, 109, 221-267.

Parlett, K. y Weston-Scheuber, K. M. (2004). Consent to Treatment for Transgender and Intersex Children. Deakin Law Review, 9, 375-394.

Peron, M. (2015). Intersexualisme, l'admission d'un troisième genre au regard des exemples étrangers. La Revue des droits de l'homme, 8, 1-6.

Peterson, A. (2010). But She Doesn't Run Like a Girl...: The Ethic of Fair Play and the Flexibility of the Binary Conception of Sex. Tulane Journal of International and Comparative law, 19, 315-335.

Puluka, A. (2015). Parent versus State: Protecting Intersex Children form Cosmetic Genital Surgery. Michigan State Law Review, 2095-2141.

Reilly, E. (2005). Radical Tweak-Relocating the Power to Assign Sex. From Enforcer of Differentiation to Facilitator of Inclusiveness: Revising the Response to Intersexuality. Cardozo Journal Law and Gender, 12, 297-335.

Reis, E. (2007). Divergence or Disorder? The politics of naming intersex. Perspectives in Biology and Medicine, 50, 535-543.

Reynal Reillo, E. (2017). Consentimiento informado y responsabilidad en el ámbito sanitario. Cizur Menor: Thomson Reuters Aranzadi.

Rodotà, S. (1995). Présentation générale des problèmes liés au transexualisme. En Transexualisme, médicine et droit, actes XXIIIe Colloque de droit européen. Strasbourg: Editions du Conseil d'Europe.

Rolker, C. (2015). Der Zwitterparagraph des ALR (1794). En Männlich-weiblich-zwischen. Disponible en: http://intersex.hypotheses.org/1194.

Sardinero García, C. (2016). Responsabilidad administrativa, civil y penal por falta de información en el ámbito clínico. Criterios indemnizatorios. Valencia: Tirant lo Blanch.

Scherpe, J. (2011). Changing One's Legal Gender In Europe. The "W" Case in Comparative Perspective. Hong Kong Law Journal, 41, 109-123.

Schmidt, D. S. (2013). Bathroom Bias: Making the Case for Trans Rights under Disability Law. Michigan Journal of Gender and Law, 20, 155-186.

Skougard, E. (2011). The Best Interests of Transgender Children. Utah Law Review, 1161-1201.

Swiss National Advisory Commission on Biomedical Ethics NEK-CNE. (2012). On the management of differences of sex development. Ethical issues relating to "intersexuality". Opinion, 20/2012. Berne. 
Tamar-Mattis, A. (2006). Exceptions to the Rule: Curing the Law's Failure to Protect Intersex Infants. Berkeley Journal of Gender, Law and Justice, 21, 59-110.

Thorn, E. D. (2014). Drop the Knife! Instituting Policies of nonsurgical intervention for intersex infants. Family Court Review, 52, 610-621.

Tompkins, A. (2014). Asterisk. Transgender Studies Quarterly, 1, 27-28.

Uslan, S. S. (2010). What Parents Don't Know: Informed Consent, Marriage, and Genital-Normalizing Surgery on Intersex Children. Indiana Law Journal, 85, 301-323.

Vanderhorst, B. (2015). Whither Lies the Self: Intersex and Transgender Individuals and A Proposal for Brain-Based Legal Sex, Harvard Law and Policy Review, 9, 241-274.

Vázquez-Pastor Jiménez, L. (2012). El transexualismo primario y su contemplación legal en el ordenamiento jurídico español. En S. Navas Navarro (dir.). Iguales y diferentes ante el Derecho privado. Valencia: Tirant lo Blanch.

Wacke, A. (1990). Del hermafroditismo a la transexualidad. Anuario de Derecho Civil, 43 (3), 677-711.

White, R. L. (2014). Preferred private parts: importing intersex Autonomy for MC v. Aaronson. Fordham International Law Journal, 37, 777-821. 